\title{
Exposure to Perflouroalkyl acids and foetal and maternal thyroid status: a review
}

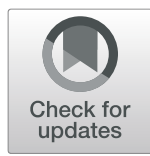

Sophie A. H. Boesen ${ }^{1}$, Manhai Long ${ }^{1}$, Maria Wielsøe ${ }^{1}$, Vicente Mustieles ${ }^{2,3}$, Mariana F. Fernandez ${ }^{2,3}$ and Eva C. Bonefeld-Jørgensen ${ }^{1,4^{*}}$ (D)

\begin{abstract}
Background: Exposure to perfluorinated-alkyl-acids (PFAAs) is ubiquitous. PFAAs are hormone-disrupting compounds that are strongly suspected to affect mother-child-health such as fetal growth. Thyroid disruption is a plausible mechanism of action. We aim to summarize the epidemiological evidence for the relation between prenatal and postnatal exposure to PFAAs and disruption of thyroid homeostasis in mothers and/or infants.

Method: Fifteen original publications on PFAAs concentrations and thyroid hormones (TH) in pregnant women and/or infants were found upon a literature search in the PubMed database. Information on exposure to seven PFAAs congeners [Perfluorooctane sulfonate (PFOS), Perfluorooctanoate (PFOA), Perfluorohexane sulfonate (PFHxS), Perfluorononanoic acid (PFNA), Perfluorodecanoic acid (PFDA), Perfluoroundecanoic acid (PFUnA), and Perfluorododecanoic acid (PFDoA)] and thyroid stimulating hormone (TSH), free and total thyroxine (FT4 and TT4), free and total triiodothyronine (FT3 and TT3), T3RU (Free triiodothyronine resin uptake) and FT4-index (FT4I) levels were recorded. We evaluated sampling of maternal TH by trimester, and infant TH by sex stratification. Reported associations between mother or infant PFAAs and TH were not uniformly assessed in the selected studies.
\end{abstract}

Results: Ten out of the fifteen studies examined maternal PFAAs concentration and TSH level. Seven studies showed significant associations between TSH and exposure to six PFAAs congeners, most of them were positive. Maternal T4 and T3 were investigated in nine studies and five studies found inverse associations between exposure to six PFAAs congeners and TH (TT3, TT4, FT3, FT4 and FT4I) levels.

Eight of the fifteen studies investigated PFAAs concentrations and infant TSH. Infant TSH level was significantly affected in four studies, positively in three studies. Nine studies investigated infant T4 and T3 and seven studies found significant associations with PFAAs exposure. However, both inverse and positive significant associations with infant TH were found eliciting no clear direction.

Conclusion: Results indicate a mainly positive relationship between maternal PFAAs concentrations and TSH levels, and suggestion of an inverse association with T4 and/or T3 levels. Associations of infant TH with PFAAs concentration were less consistent.

Keywords: Perfluorinated-alkyl-acids, PFAAs, Thyroid hormones, TSH, Maternal, Infant, Mother-child-health, Human biomonitoring, HBM4EU

\footnotetext{
* Correspondence: ebj@ph.au.dk

'Centre for Arctic Health and Molecular Epidemiology, Department of Public

Health, Aarhus University, Aarhus, Denmark

${ }^{4}$ Greenland Centre for Health Research, University of Greenland, Nuuk,

Greenland

Full list of author information is available at the end of the article
}

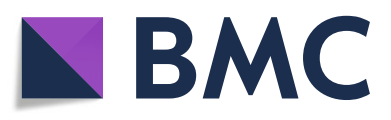

(c) The Author(s). 2020 Open Access This article is licensed under a Creative Commons Attribution 4.0 International License, which permits use, sharing, adaptation, distribution and reproduction in any medium or format, as long as you give appropriate credit to the original author(s) and the source, provide a link to the Creative Commons licence, and indicate if changes were made. The images or other third party material in this article are included in the article's Creative Commons licence, unless indicated otherwise in a credit line to the material. If material is not included in the article's Creative Commons licence and your intended use is not permitted by statutory regulation or exceeds the permitted use, you will need to obtain permission directly from the copyright holder. To view a copy of this licence, visit http://creativecommons.org/licenses/by/4.0/. The Creative Commons Public Domain Dedication waiver (http://creativecommons.org/publicdomain/zero/1.0/) applies to the data made available in this article, unless otherwise stated in a credit line to the data. 


\section{Background}

Increasing evidence suggests that in utero exposure to perfluorinated-alkyl-acids (PFAAs), a subgroup of persistent organic pollutants (POPs), have adverse effects on fetal growth and development [1]. Exposure to PFAAs has also been shown to affect the thyroid function [2-4], decrease vaccination immune response in children [5, 6], increase the risk of breast cancer [7], and the development of metabolic syndrome, type 2 diabetes [8], lipoedema and cardiovascular disease $[1,9,10]$.

PFAAs are detected globally in human populations and wildlife [11-13]. Humans are mainly exposed to PFAAs through food items and drinking water, but also by inhalation and dermal exposure [14]. The mean halflife of serum elimination in humans is 5.4 years for perfluorooctane sulfonate (PFOS), 3.8 years for perfluorooctanoate (PFOA) and 8.5 years for perfluorohexane sulfonate (PFHxS) [15]. Since 2000, several western countries have made restrictions on the PFAAs production [16], but presently only PFOS and PFOA are regulated by the Stockholm Convention on Persistent Organic Pollutants [17]. Thus, human serum concentrations, especially for the regulated PFOS and to some degree PFOA, have decreased during the last decade $[18,19]$.

PFAAs enter the foetal circulation through placenta transfer and postnatally the infant is exposed by breastfeeding [20]. Thus, the foetus is exposed to PFAAs at highly vulnerable developmental stages [21]. Disruption of hormonal pathways involving the hypothalamicpituitary-thyroid (HPT) and hypothalamic-pituitarygonadal (HPG) axes has been hypothesized as a mechanism of action (MoA) and mediating steps between the effects of PFAAs on foetal growth [22]. Maternal abnormal thyroid function during pregnancy might affect foetal growth and neural development [23-28]. The foetus' thyroid hormone (TH) production does not suffice until midgestation but rely on maternal supply mainly during the 1st trimester, implying a high demand on the maternal HPT axis [23].

Maternal thyroxine (T4) and triiodothyronine (T3) hormones crosses the placenta, but not the maternal thyroid stimulating hormone (TSH) [29, 30]. In the pregnant woman, the $\mathrm{TH}$ production undergoes dynamic and physiological changes: TSH decreases during the 1st trimester and stabilizes in the 2nd and 3rd trimester [31]. The European Thyroid Association guidelines recommend a TSH upper reference limit of 2.5 $\mathrm{mIU} / \mathrm{L}$ in the 1 st trimester and $3.0 \mathrm{mU} / \mathrm{L}$ in the second and third trimesters [32], though it is discussed whether the limits are too narrow [31]. In the absence of preexisting thyroid disease, the American Thyroid Association guideline recommends upper limit of $4 \mathrm{mIU} / \mathrm{L}$ in early pregnancy week 7-12 and gradually during 2 nd and 3rd trimesters a return to non-pregnancy range
[33]. High human corionic gonadotropin possess a thyrotrophic effect and elicit a physiological increase in levels of T4 and T3. High oestrogen levels in pregnancy increase circulating levels of thyroxin binding globulin (TBG), and may lead to an increase in the total level of T4 and T3 [34, 35]. The thyroid binding capacity is facilitated by several plasma proteins such as TBG, transthyretin (TTR) and albumin [36]. Thyroid peroxidase (TPO) is a key enzyme of TH biosynthesis in the thyroid gland [37]. Thyroid peroxidase-antibodies (TPOAb) may potentially be an important covariate. TPOAb production during pregnancy might be indicative of thyroid stress especially when levels are above a cut-off value [26].

This review will focus on exposure to PFAAs and thyroid hormone homeostasis in the mother and infant. Effect estimates were evaluated with the objective to understand whether PFAAs exposure is related to hypothyroidism (low free T4, high TSH) or hyperthyroidism (high free $\mathrm{T} 4$, low $\mathrm{TSH}$ ).

\section{Method}

The PubMed database was searched, using Medical Subject Headings (MESH) terms and specific keywords, to locate relevant publications, following a specific strategy (Table 1). Last literature search was conducted on the 21th January 2020. Citations from selected publications were also investigated. This report was restricted to epidemiological studies with a sample size $>100$ participants. All selected studies are summarized in Tables 2, $3,4,5,6,7,8,9$, and 10 .

\section{Data extraction}

Following data was extracted to supply general information and study characteristics (Table 2): Study design and population, year of enrollment, number of participants, exclusion criteria, biological matrix for exposure assessment, pregnancy trimester of exposure assessment and $\mathrm{TH}$ outcome measurement, PFAA compounds examined above the limits of detection/quantification (LOD/LOQ), outcome estimates and confounders.

Tables 3 and 4 give the information on exposure, including trimester of PFAAs measurement, participants, median concentration $(\mathrm{ng} / \mathrm{mL})$ and $\%<\mathrm{LOD} / \mathrm{LOQ}$. Tables 5 and 6 give the maternal and infant TH levels, respectively. Tables 7, 8, 9 and 10 show the original data on thyroid hormone outcome upon PFAAs exposure.

\section{Results}

\section{Study populations}

We found 15 original publications that fulfilled the inclusion criteria [38-52] (Table 2). Study populations varied from 118 to 1366 participants [40,51], conducted in Asia $(n=6)$, Europe $(n=6)$ and Northern America $(n=3)$. Study designs were cross sectional and/or cohort studies. 
Table 1 Literature search strategy

\begin{tabular}{|c|c|c|c|}
\hline $\begin{array}{l}\text { Database } \\
\text { Pubmed, sorted by "Best Match" }\end{array}$ & \multicolumn{2}{|c|}{ MESH and non-MESH terms } & \multirow{2}{*}{$\begin{array}{l}\text { Hits } \\
17,054\end{array}$} \\
\hline Pubmed, sorted by "Best Match" & & $\begin{array}{l}\text { (Perfluorinated OR Perfluorinated acid OR perfluorooctane sulfonic acid OR } \\
\text { perfluorooctanoic acid OR fluorocarbons OR polyfluorinated OR polyfluoroalkyl } \\
\text { OR perfluoroalkyl Perfluorochemicals OR PFOS OR PFOA OR PFNA OR PFDA } \\
\text { OR PFHxS OR PFUnA OR PFOSA OR PFDA) }\end{array}$ & \\
\hline & AND & $\begin{array}{l}\text { (Thyroid OR Thyroid hormones OR thyroxine OR triiodothyronine OR } \\
\text { thyroid-stimulating hormone OR TSH OR T4 OR T3) }\end{array}$ & 223 \\
\hline & AND & (Pregnancy OR maternal OR mother OR newborn OR infant OR cord) & 59 \\
\hline \multirow[t]{2}{*}{ Studies full filling inclusion criteria } & Participants & $\begin{array}{l}\text { Pregnant women and/or infants with measurements of PFAAs exposure } \\
\text { and thyroid hormones. }\end{array}$ & 20 \\
\hline & Number & > 100 participants. & 15 \\
\hline
\end{tabular}

Most studies adjusted for maternal age and/or parity (Table 2).

\section{PFAAs exposure measurement}

Tables 3 and 4 show the PFAAs level in mothers and infants, respectively. PFAAs exposure was assessed in maternal plasma or serum (10 studies, Tables 2 and 3 ) collected in 1st trimester $(n=2)[39,40]$, 2nd trimester $(n=5)[38,41-43,45]$ or 3rd trimester $(n=4)$ [38, 44, $46,47]$. One study measured the maternal PFAAs level between 24 and 41 weeks of gestational age (2nd, 3rd trimester) or within 5 days after delivery [38]. PFAAs were also assessed in cord blood serum / plasma $(n=6)[46$, 48-52] (Tables 2 and 4).

In all studies, PFAAs were measured using liquid chromatography mass-spectrometry techniques. We have in this review focused on seven PFAAs congeners ((n) studies): PFOS $(n=15)$, PFOA $(n=15)$, PFHxS $(n=12)$, PFNA $(n=13)$, PFDA $(n=10)$, PFUnA $(n=10)$, and PFDoA $(n=$ $6)$, although the studies also examined other congeners [PFHpS, EtFOSAA, MeFOSAA, 6:2 FTS, NEtFOSAA, NMeFOSAA, PFPeA, PFHpA, PFTrDA, PFTeDA and PFOS isomers], which were not included in this review. The range of maternal PFOS median concentration was $4.4 \mathrm{ng} / \mathrm{mL}$ [46] to $29.5 \mathrm{ng} / \mathrm{mL}$ [40] (Table 3). Among infants, the range of the PFOS concentration was $0.66 \mathrm{ng} /$ $\mathrm{mL}$ [49] to $5.11 \mathrm{ng} / \mathrm{mL}$ [51] in umbilical cord blood serum/plasma (UCB) samples (Table 4).

\section{Thyroid hormone assessment}

The measurement of thyroid hormones varied between the studies, showing mainly TSH, free T4 (FT4), total T4 (TT4), free T3 (FT3) and total T3 (TT3) levels or variations hereof, i.e. FT4 index (FT4I), calculated from TT4 and T3-resin uptake $(\mathrm{FT} 4 \mathrm{I}=\mathrm{TT} 4 \times \mathrm{T} 3$ resin uptake) $[39,47]$.

Maternal TH were measured during the 1 st trimester $(n=3)$ [38-40], 2nd trimester $(n=2)$ [41, 42], 3rd trimester $(n=3)[44,46,47]$ or at multiple time points $(n=2)[43,45]$. Infant TH were assessed postpartum in cord blood serum/plasma $(n=6)[44,46,47,49,51,52]$ or infant serum obtained at heel stick up to 7 days postpartum $(n=4)[38,39,48,50]$ (Table 2).

Thus, maternal PFAAs exposure and maternal $\mathrm{TH}$ levels were predominantly measured simultaneously with exception of Kato et al. who measured maternal TH in the 1st trimester, while PFAAs exposure was measured after 1st trimester [38] (Table 2).

Tables 5 and 6 shows the maternal and infant TH levels, respectively. The maternal TSH median levels were in general lower than the upper reference limit with no obvious trimester differences. In general similar TT3 and FT3 levels were observed among the reviewed studies, as expected with lower FT3 $(\mathrm{pmol} / \mathrm{L})$ levels than the TT3 $(\mathrm{nmol} / \mathrm{L})$ levels. Also the FT4 and TT4 levels were in overall similar among the studies with significantly higher TT4 levels (Table 5). Four studies [39, 42, 43, 45] also determined the maternal TPOAb finding positive levels.

As shown in Table 6, infant TSH median levels were in general within the normal reference range (i.e. being higher than the lower limit but lower than the upper limit). Moreover, as expected for the T3 (TT3 and FT3), the TT3 levels were higher than FT3. Similar values of T4 (TT4 and FT4) levels were also seen among the studies and FT4 levels were mainly within the lower limit range (Table 6).

\section{Association between PFAAs exposure and thyroid hormone outcomes}

The associations between PFAAs exposure and $\mathrm{TH}$ outcomes are outlined by the following order: i) maternal TSH (Table 7), ii) maternal T4 and T3 (Table 8), iii) infant TSH (Table 9) and iv) infant T4 and T3 (Table 10). Maternal TH estimates were assessed by the trimester in which they were measured. Infant $\mathrm{TH}$ estimates were assessed upon stratification by sex (Suppl. Tables 1 and 2).

\section{Maternal PFAAs exposure and TSH levels (Table 7)}

Two out of nine studies (Table 7) showed a significantly positive association between maternal PFHxS concentrations and TSH levels in the 3rd trimester 


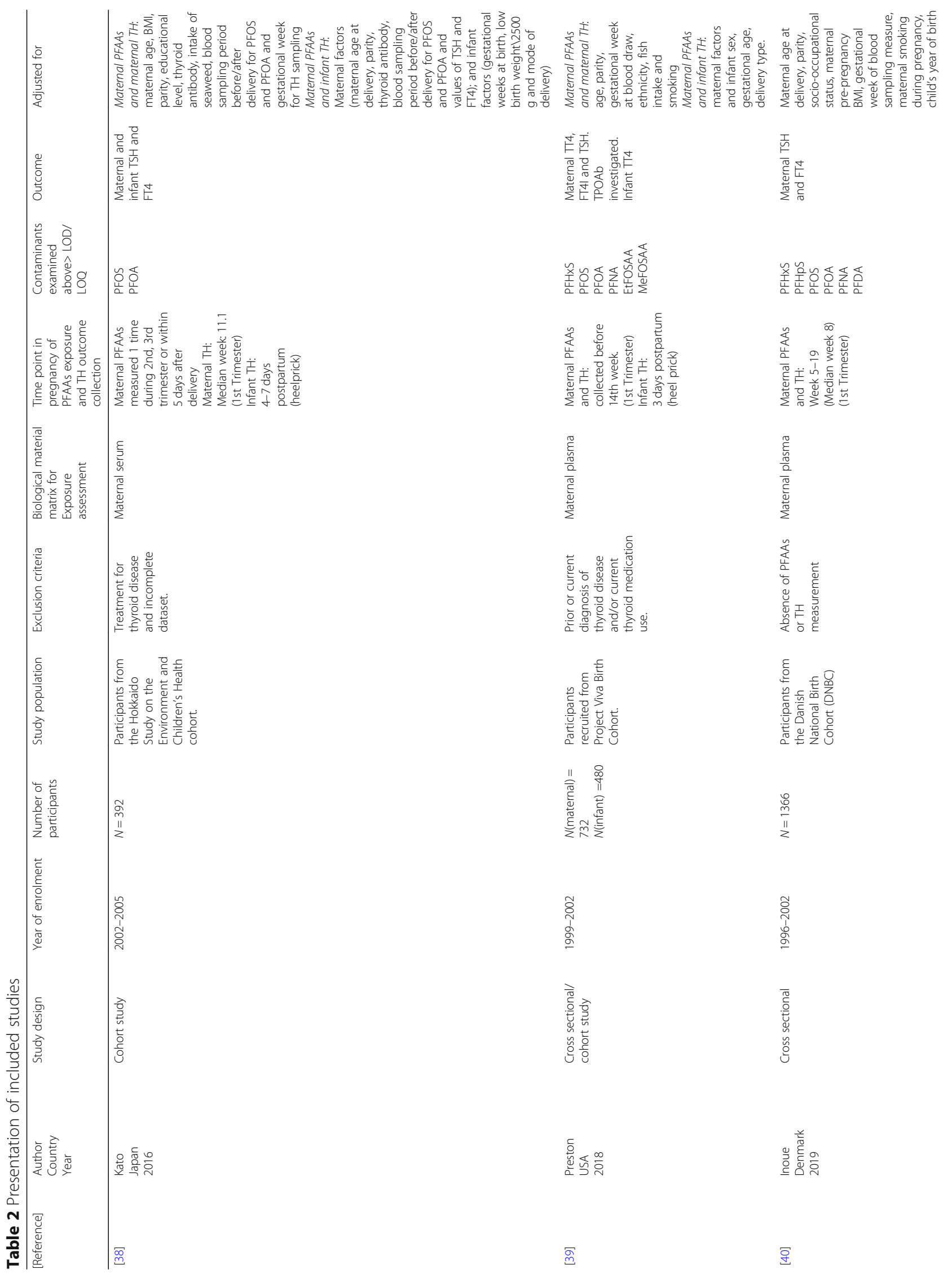




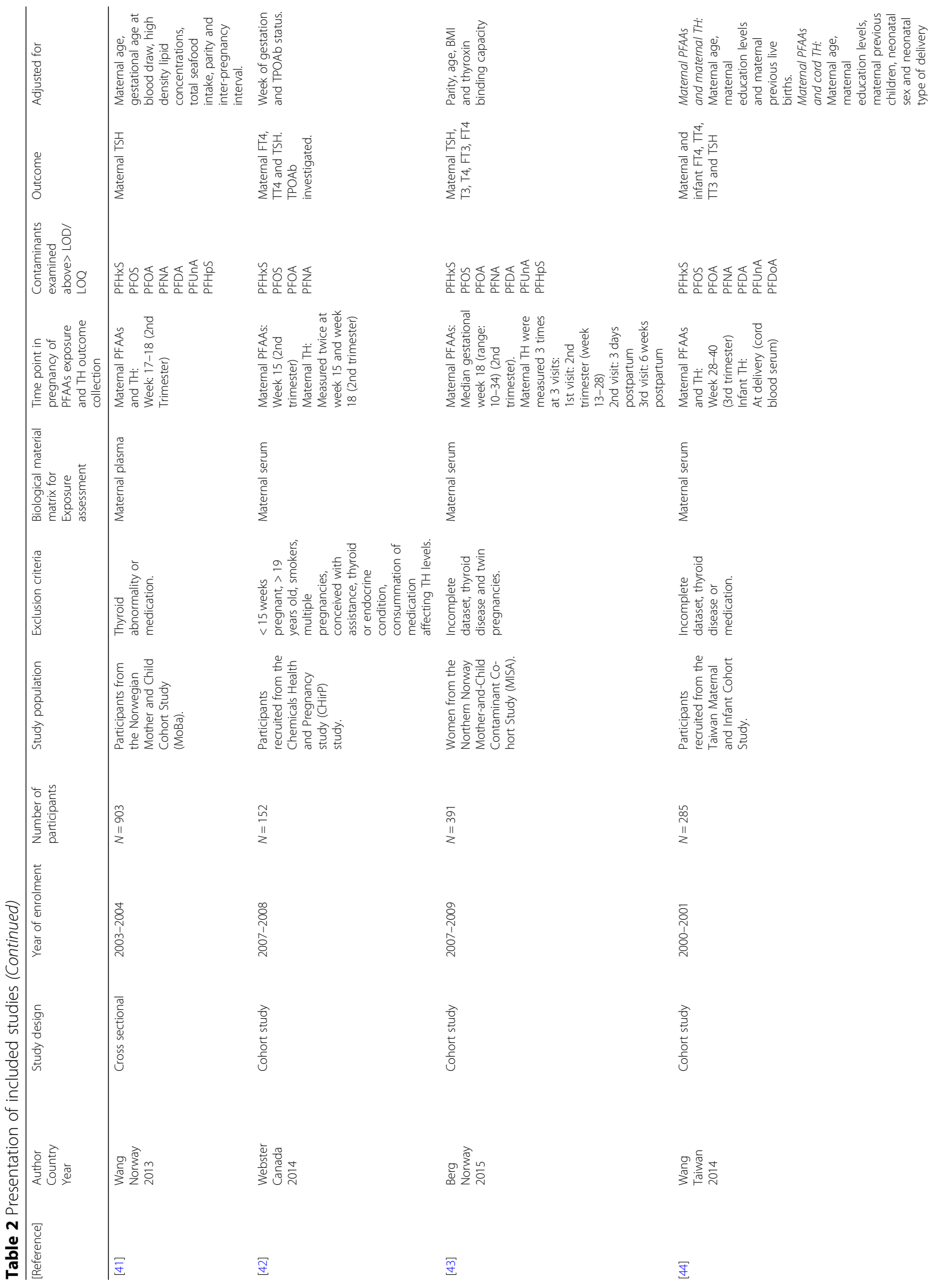




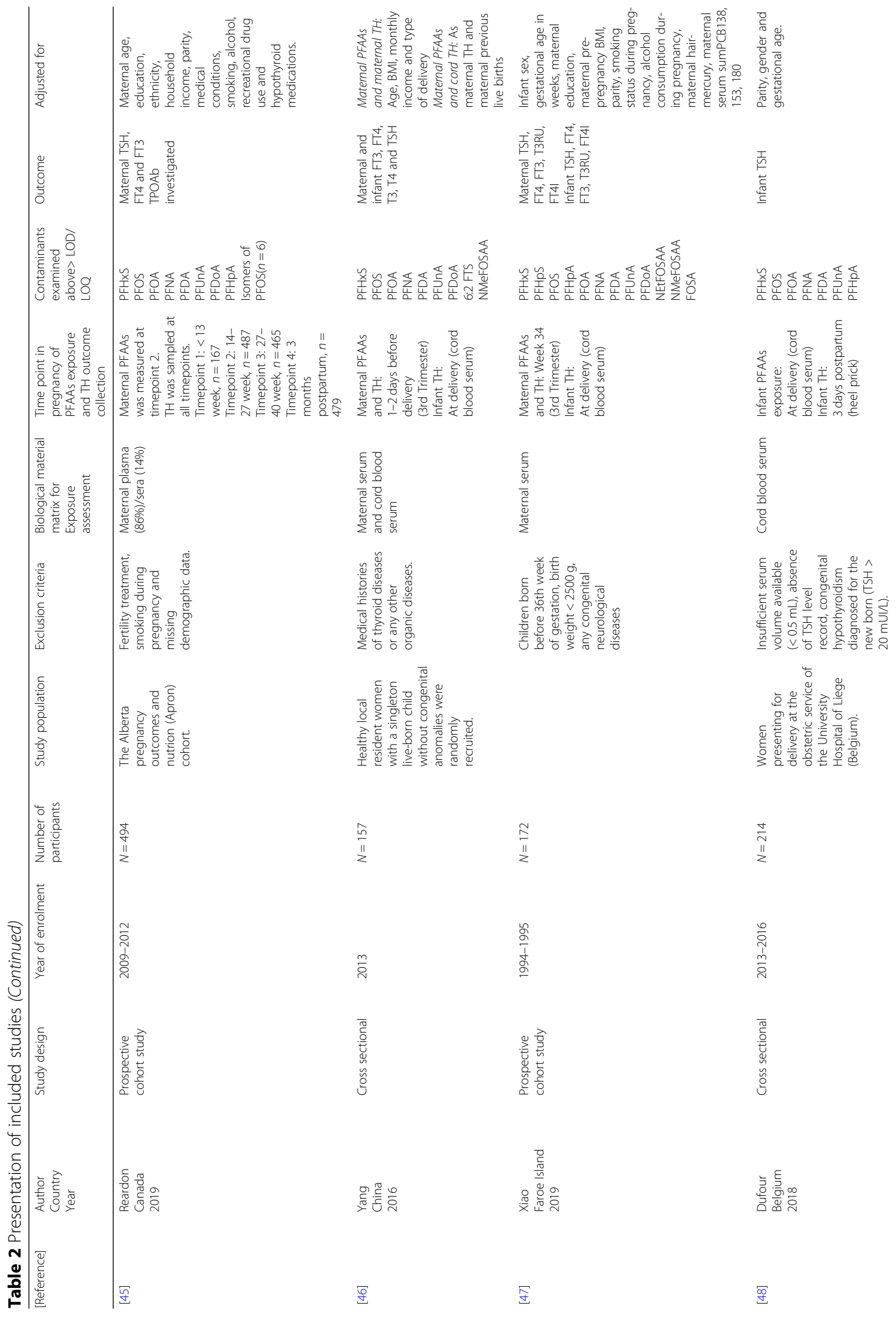




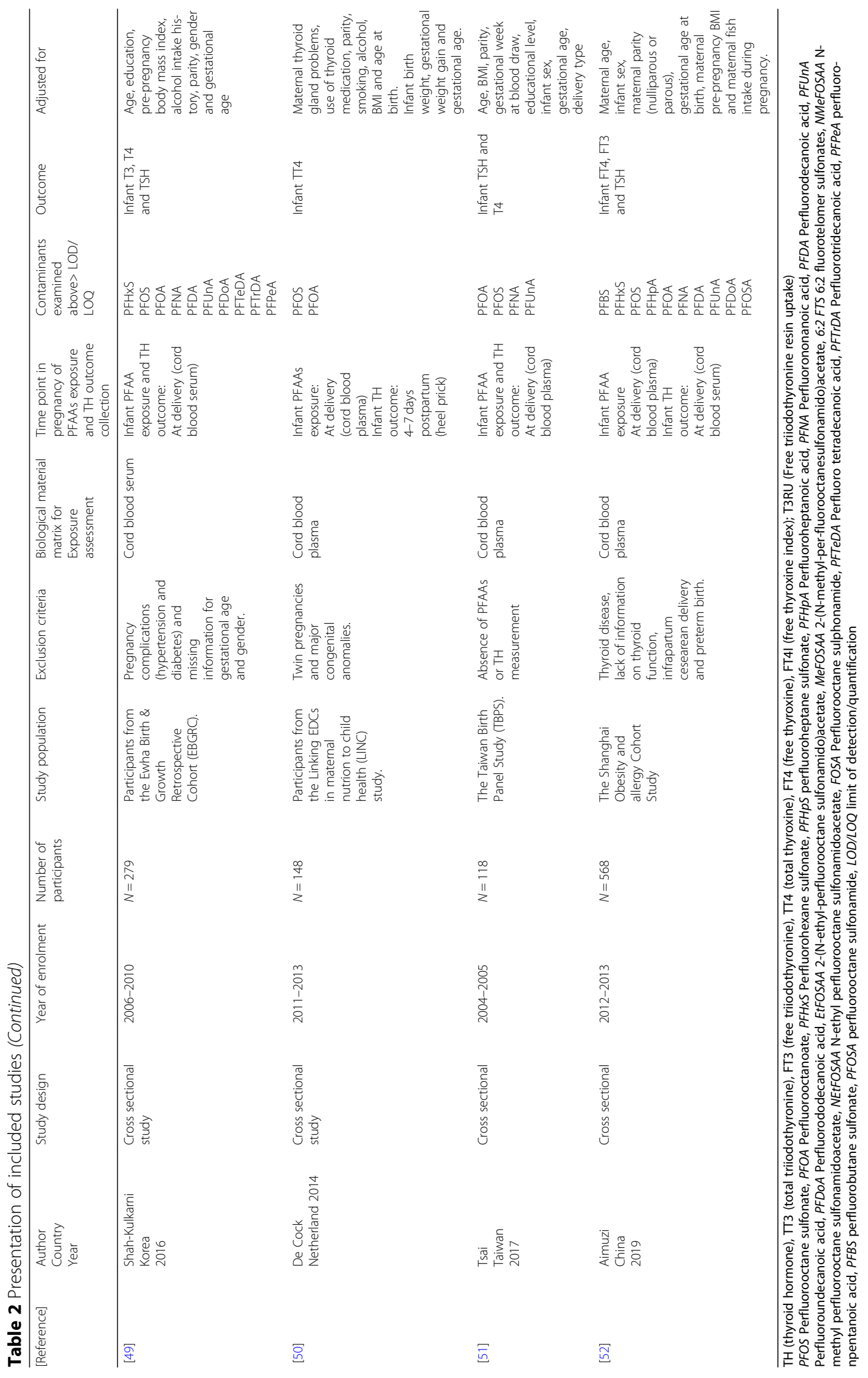


Boesen et al. Environmental Health $\quad$ (2020) 19:107

Page 8 of 28

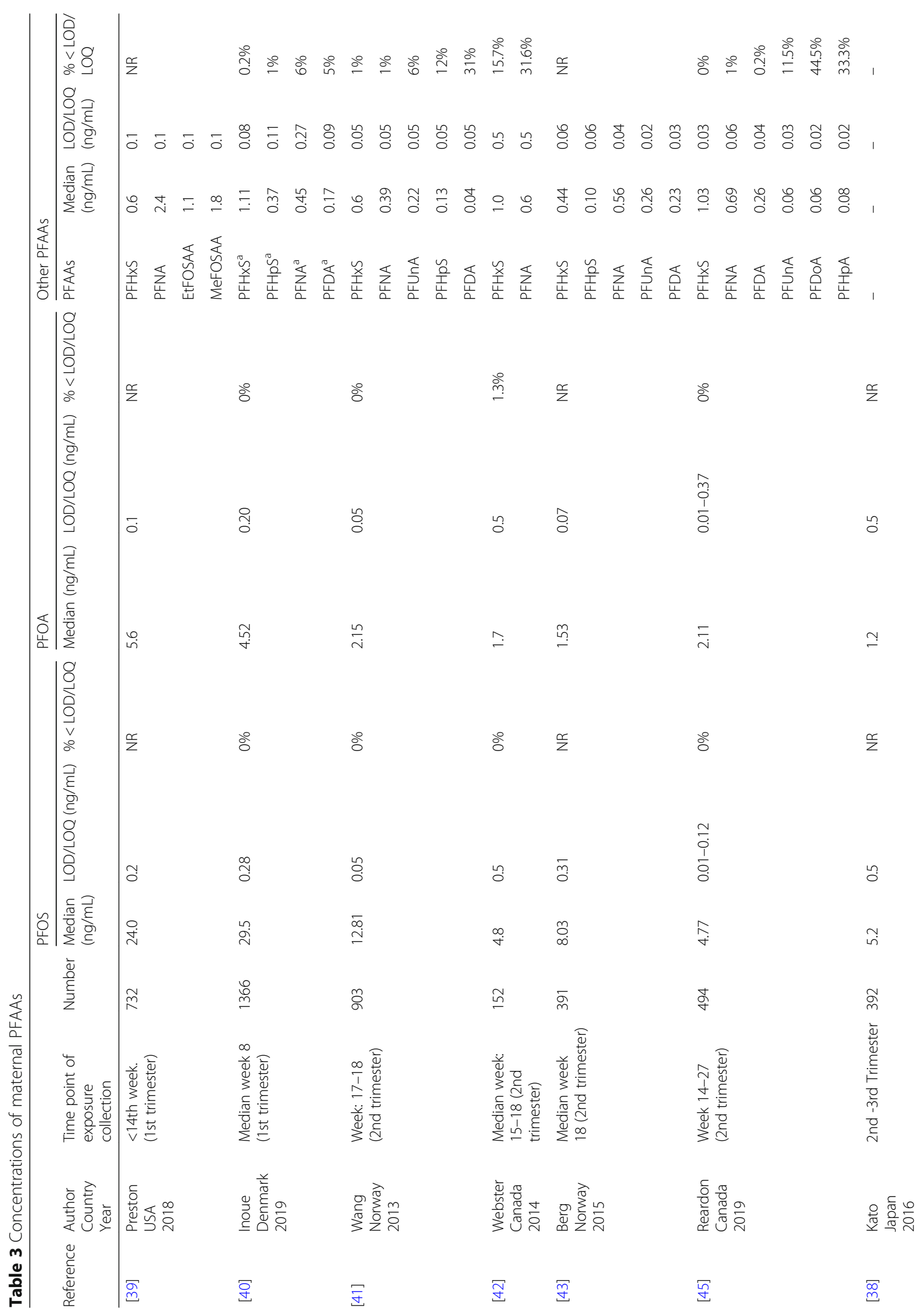




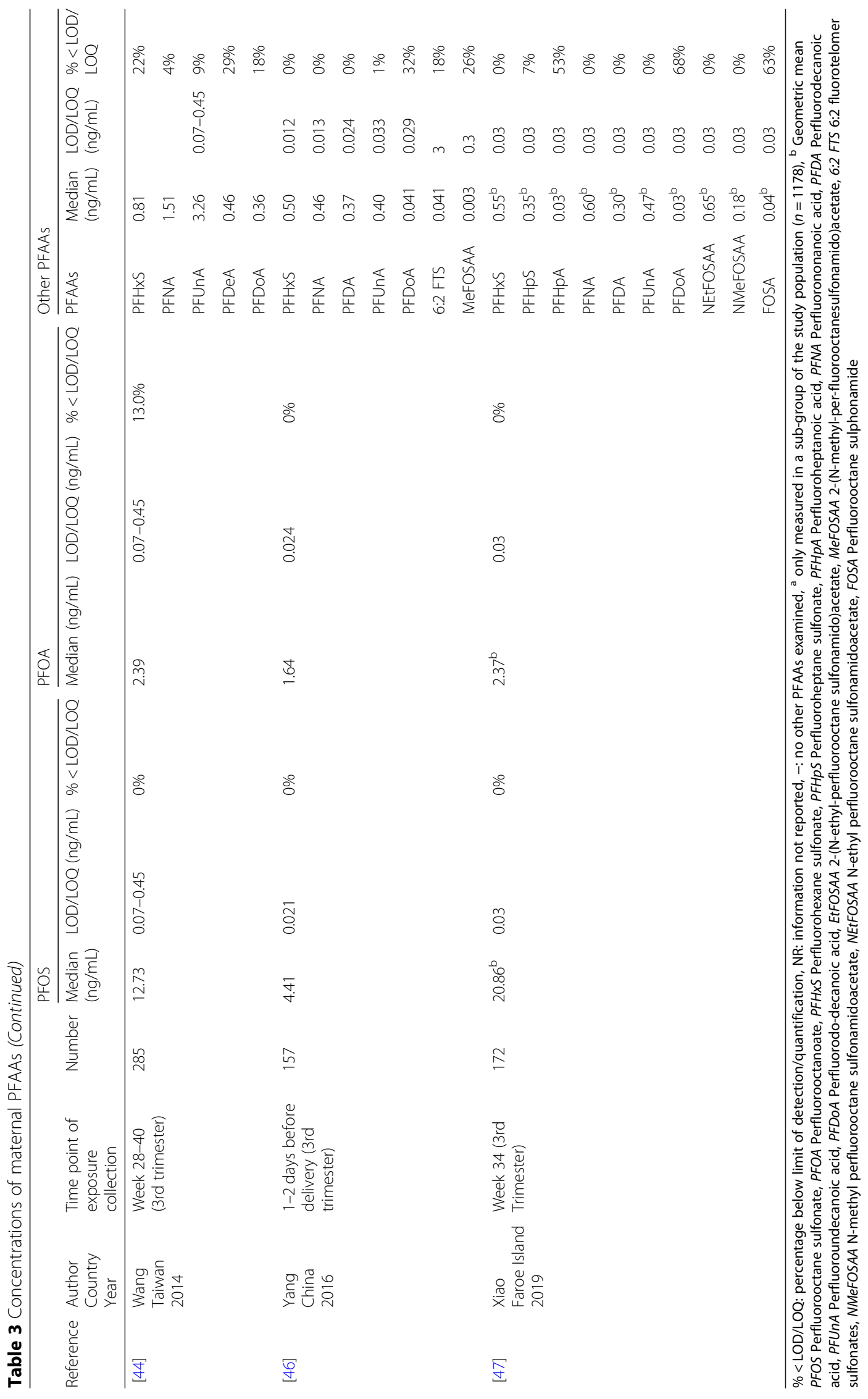




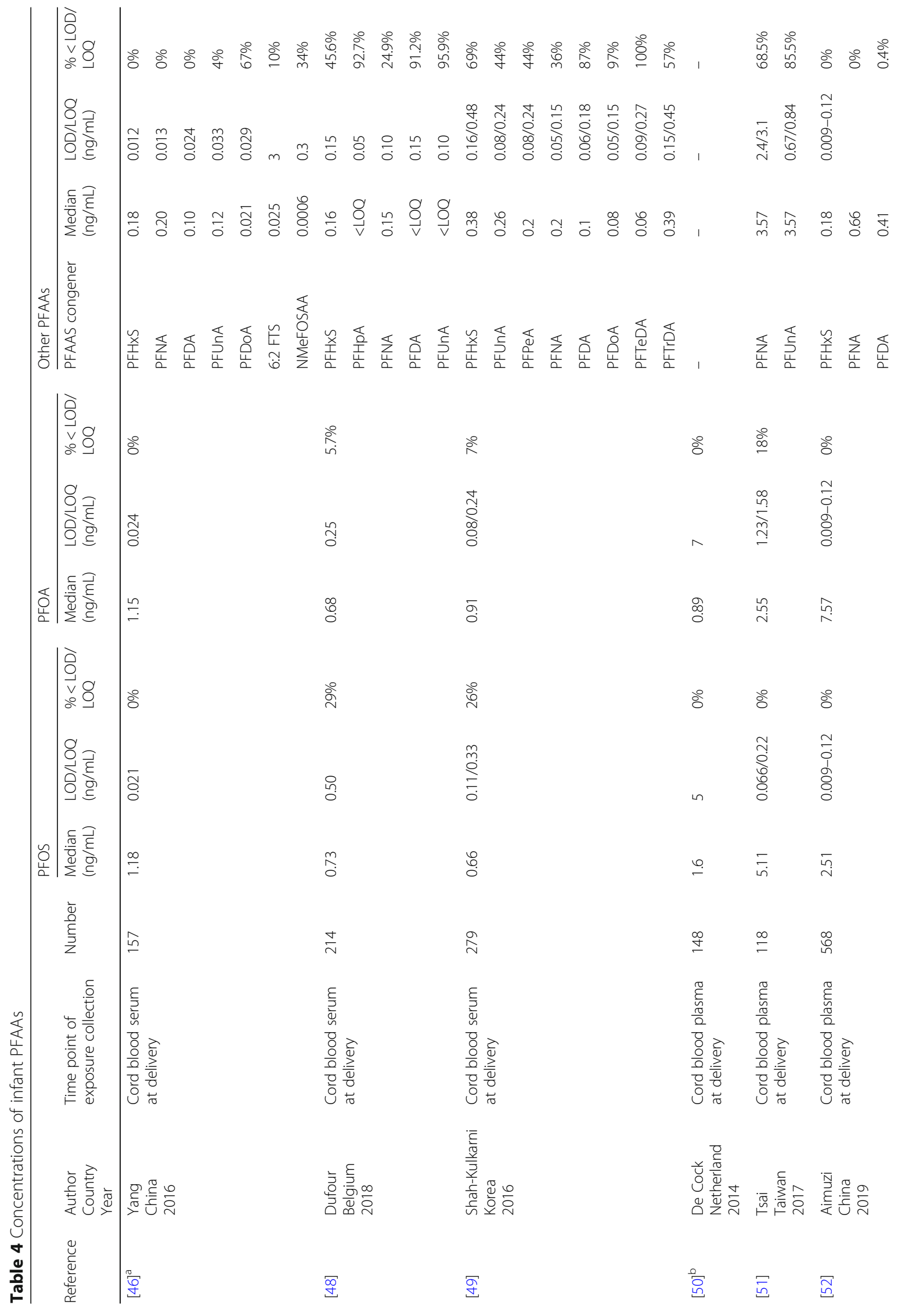




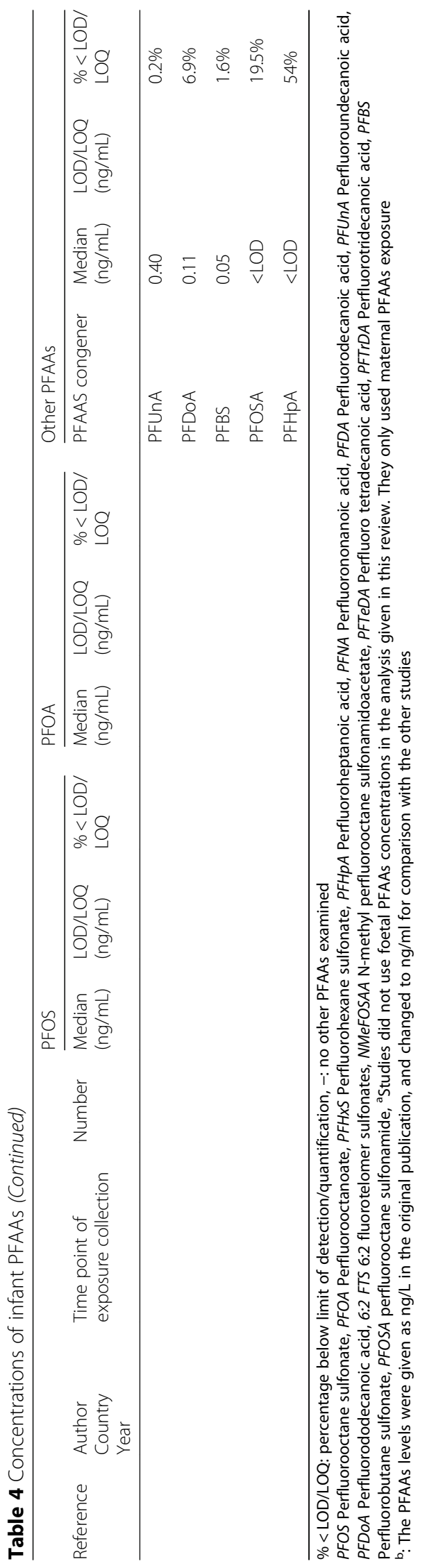




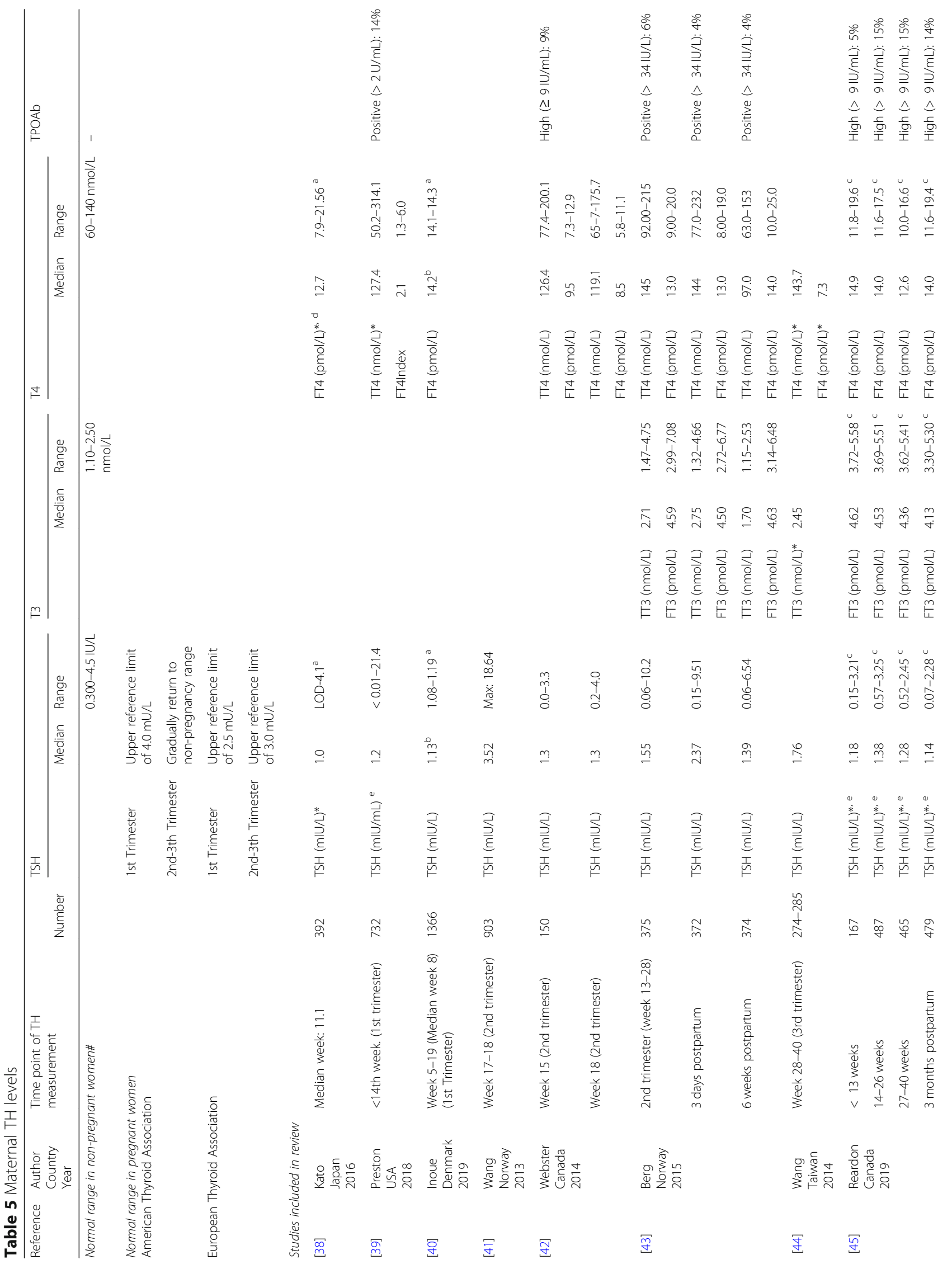




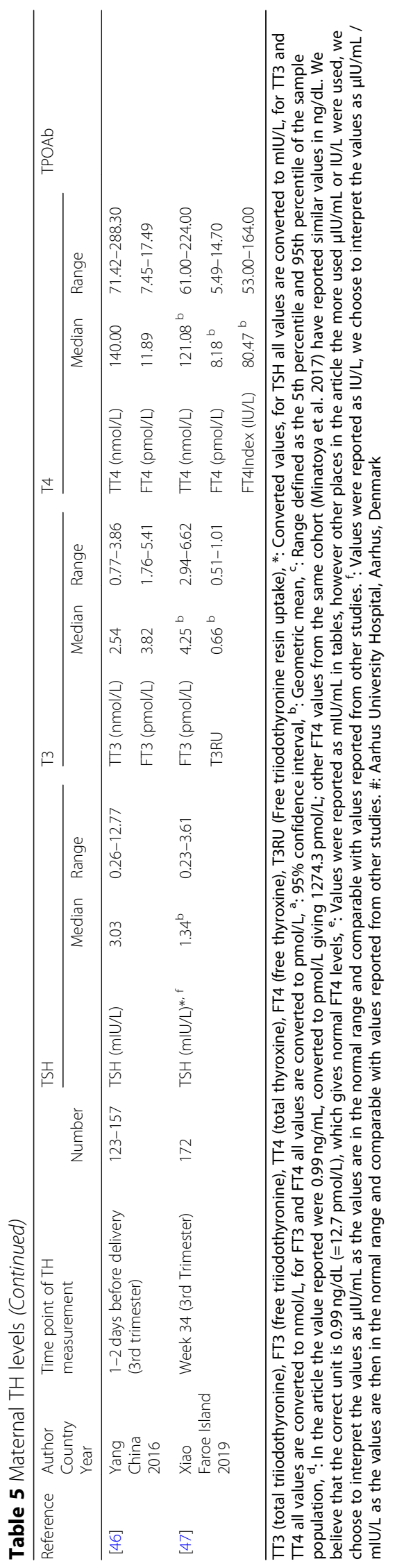




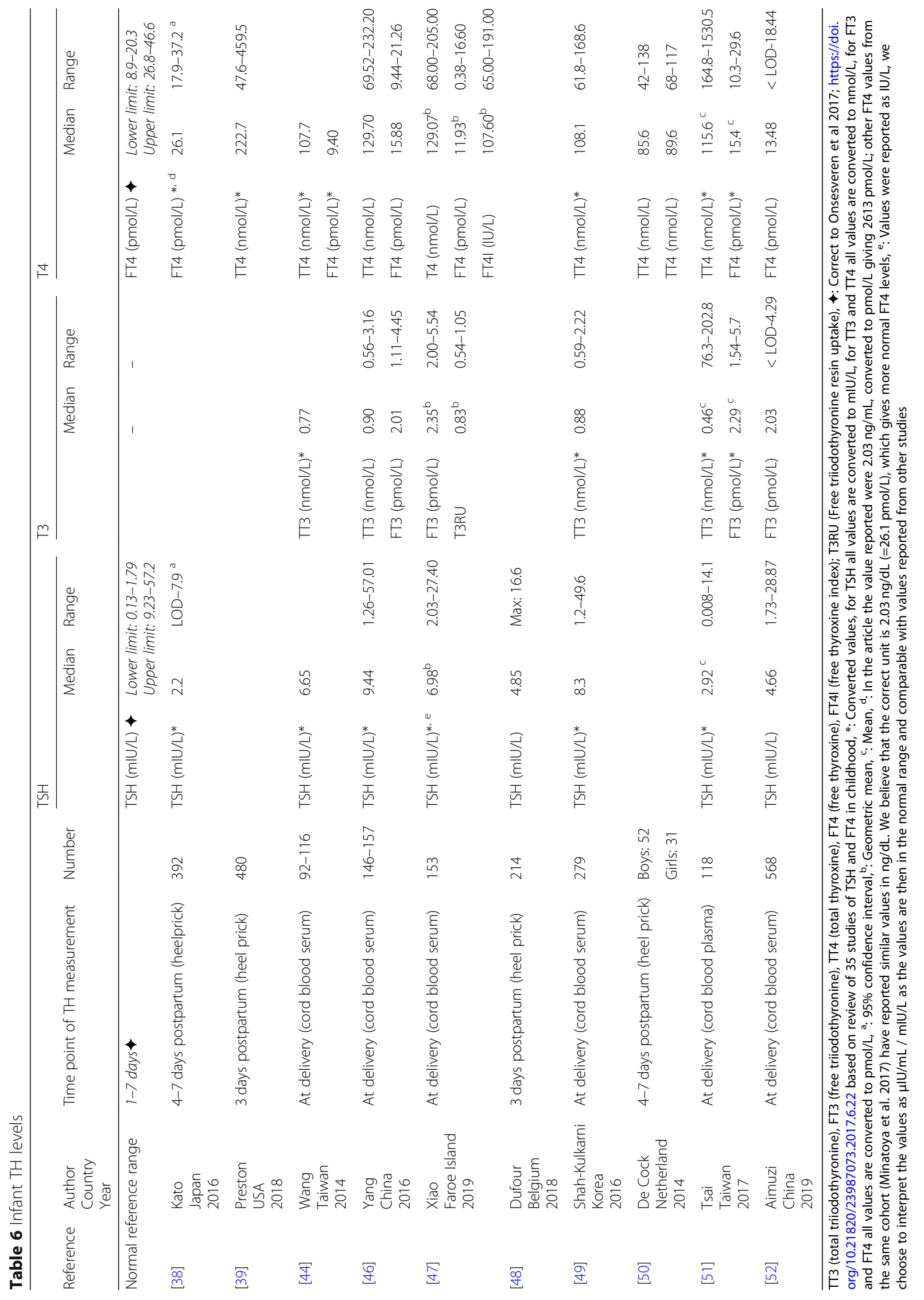




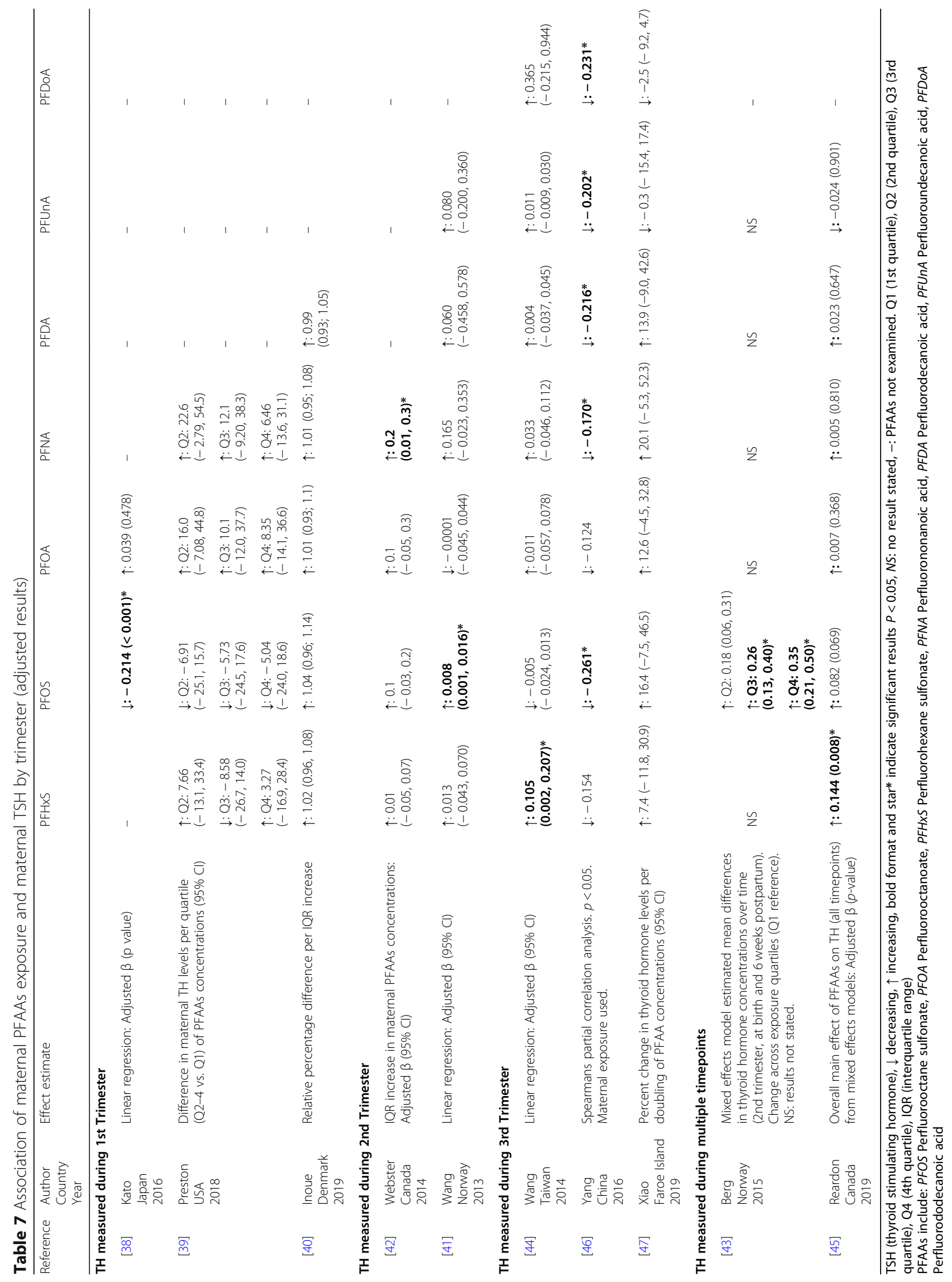


Table 8 Association of maternal PFAAs exposure and maternal T4 and T3 by trimester (adjusted results)

\begin{tabular}{|c|c|c|c|c|c|c|c|c|c|c|}
\hline Reference & $\begin{array}{l}\text { Author } \\
\text { Country } \\
\text { Year }\end{array}$ & Effect estimate & TH & $\mathrm{PFH} \times \mathrm{S}$ & PFOS & PFOA & PFNA & PFDA & PFUnA & PFDoA \\
\hline \multicolumn{11}{|c|}{ TH measured during 1st Trimester } \\
\hline [38] & $\begin{array}{l}\text { Kato } \\
\text { Japan } \\
2016\end{array}$ & $\begin{array}{l}\text { Linear regression: Adjusted } \beta \\
(p \text { value) }\end{array}$ & FT4 & - & $\begin{array}{l}\uparrow: 0.061 \\
(0.236)\end{array}$ & $\begin{array}{l}\uparrow: 0.004 \\
(0.946)\end{array}$ & - & - & - & - \\
\hline \multirow[t]{6}{*}{ [39] } & \multirow[t]{6}{*}{$\begin{array}{l}\text { Preston } \\
\text { USA } \\
2018\end{array}$} & \multirow[t]{6}{*}{$\begin{array}{l}\text { Difference in maternal TH levels } \\
\text { per quartile (Q2-4 vs. Q1) of } \\
\text { PFAA concentrations ( } 95 \% \text { Cl) }\end{array}$} & \multirow[t]{3}{*}{ FT4lC } & $\begin{array}{l}\downarrow \text { Q2: }-0.25 \\
(-3.55,3.16)\end{array}$ & $\begin{array}{l}\downarrow \mathrm{Q} 2:-0.56 \\
(-3.93,2.93)\end{array}$ & $\begin{array}{l}\downarrow Q 2: \\
-4.68 \\
(-7.93 \\
-1.32)^{*}\end{array}$ & $\begin{array}{l}\downarrow \mathrm{Q} 2:-1.94 \\
(-5.48,1.72)\end{array}$ & - & - & - \\
\hline & & & & $\begin{array}{l}\downarrow \text { L } 3:-0.35 \\
(-3.77,3.18)\end{array}$ & $\begin{array}{l}\downarrow \text { ¿ 3: }-1.41 \\
(-4.80,2.09)\end{array}$ & $\begin{array}{l}\downarrow \text { Q3: }-2.89 \\
(-6.23,0.58)\end{array}$ & $\begin{array}{l}\downarrow Q 3:-0.53 \\
(-3.79,2.85)\end{array}$ & - & - & - \\
\hline & & & & $\begin{array}{l}\downarrow \text { Q4: }-4.19 \\
(-7.43 \\
-0.85)^{*}\end{array}$ & $\begin{array}{l}\downarrow \mathrm{Q} 4:-1.51 \\
(-4.92,2.02)\end{array}$ & $\begin{array}{l}\downarrow \mathrm{Q} 4: \\
-6.52 \\
(-9.86 \\
-3.06)^{*}\end{array}$ & $\begin{array}{l}\downarrow \text { Q4: }-1.06 \\
(-4.27,2.25)\end{array}$ & - & - & - \\
\hline & & & \multirow[t]{3}{*}{$\pi 4$} & $\begin{array}{l}\downarrow \mathrm{Q} 2:-0.23 \\
(-0.61,0.14)\end{array}$ & $\begin{array}{l}\uparrow Q 2: 0.28 \\
(-0.10,0.66)\end{array}$ & $\begin{array}{l}\downarrow \text { Q2: }-0.12 \\
(-0.51,0.27)\end{array}$ & $\begin{array}{l}\uparrow Q 2: 0.02 \\
(-0.39,0.43)\end{array}$ & - & - & - \\
\hline & & & & $\begin{array}{l}\uparrow Q 3: 0.10 \\
(-0.28,0.49)\end{array}$ & $\begin{array}{l}\uparrow Q 3: 0.14 \\
(-0.25,0.52)\end{array}$ & $\begin{array}{l}\uparrow Q 3: 0.01 \\
(-0.38,0.40)\end{array}$ & $\begin{array}{l}\uparrow Q 3: 0.08 \\
(-0.29,0.45)\end{array}$ & - & - & - \\
\hline & & & & $\begin{array}{l}\downarrow \text { Q4: }-0.34 \\
(-0.73,0.04)\end{array}$ & $\begin{array}{l}\uparrow Q 4: 0.31 \\
(-0.08,0.70)\end{array}$ & $\begin{array}{l}\uparrow \text { Q4: } 0.02 \\
(-0.39,0.43)\end{array}$ & $\begin{array}{l}\downarrow \text { Q4: }-0.09 \\
(-0.45,0.28)\end{array}$ & - & - & - \\
\hline [40] & $\begin{array}{l}\text { Inoue } \\
\text { Denmark } \\
2019\end{array}$ & $\begin{array}{l}\text { Relative percentage difference } \\
\text { per IQR increase }\end{array}$ & FT4 & $\begin{array}{l}\uparrow 1.00 \\
(0.98,1.01)\end{array}$ & $\begin{array}{l}\uparrow 1.00 \\
(0.99,1.02)\end{array}$ & $\begin{array}{l}\uparrow 1.01 \\
(0.99,1.02)\end{array}$ & $\begin{array}{l}\uparrow 1.00 \\
(0.99 ; 1.01)\end{array}$ & $\begin{array}{l}\uparrow 1.01 \\
(1.00 ; 1.02)\end{array}$ & & \\
\hline \multicolumn{11}{|c|}{ TH measured during 2nd Trimester } \\
\hline [42] & $\begin{array}{l}\text { Webster } \\
\text { Canada } \\
2014\end{array}$ & $\begin{array}{l}\text { IQR increase in maternal PFAA } \\
\text { concentrations: Adjusted } \beta \\
(95 \% \mathrm{Cl})\end{array}$ & FT4 & $\begin{array}{l}\downarrow:-0.02 \\
(-0.1,0.07)\end{array}$ & $\begin{array}{l}\uparrow: 0.03 \\
(-0.2,0.2)\end{array}$ & $\begin{array}{l}\downarrow:-0.06 \\
(-0.3,0.2)\end{array}$ & $\begin{array}{l}\downarrow:-0.03 \\
(-0.2,0.2)\end{array}$ & - & - & - \\
\hline \multicolumn{11}{|c|}{$\mathrm{TH}$ measured during $3 \mathrm{rd}$ Trimester } \\
\hline \multirow[t]{3}{*}{ [44] } & \multirow[t]{3}{*}{$\begin{array}{l}\text { Wang } \\
\text { Taiwan } \\
2014\end{array}$} & \multirow[t]{3}{*}{$\begin{array}{l}\text { Linear regression: Adjusted } \beta \\
(95 \% \mathrm{Cl})\end{array}$} & FT4 & $\begin{array}{l}\downarrow:-0.010 \\
(-0.023,0.003)\end{array}$ & $\begin{array}{l}\uparrow: 0.001 \\
(-0.002 \\
0.003)\end{array}$ & $\begin{array}{l}\downarrow:-0.003 \\
(-0.012 \\
0.005)\end{array}$ & $\begin{array}{l}\downarrow:-0.019 \\
(-0.028 \\
-0.009)^{*}\end{array}$ & $\begin{array}{l}\downarrow:-0.001 \\
(-0.006 \\
0.005)\end{array}$ & $\begin{array}{l}\downarrow:-0.004 \\
(-0.007 \\
-0.002)^{*}\end{array}$ & $\begin{array}{l}\downarrow:-0.132 \\
(-0.204 \\
-0.059)^{*}\end{array}$ \\
\hline & & & $\Pi 4$ & $\begin{array}{l}\downarrow:=0.130 \\
(-0.316,0.057)\end{array}$ & $\begin{array}{l}\uparrow: 0.019 \\
(-0.016 \\
0.053)\end{array}$ & $\begin{array}{l}\uparrow: 0.011 \\
(-0.108 \\
0.130)\end{array}$ & $\begin{array}{l}\downarrow:-0.189 \\
(-0.333 \\
-0.046)^{*}\end{array}$ & $\begin{array}{l}\uparrow: 0.047 \\
(-0.028 \\
0.123)\end{array}$ & $\begin{array}{l}\downarrow:-0.062 \\
(-0.097 \\
-0.026)^{*}\end{array}$ & $\begin{array}{l}\downarrow:-1.742 \\
(-2.785 \\
-0.700)^{*}\end{array}$ \\
\hline & & & $\pi 3$ & $\begin{array}{l}\downarrow:=0.002 \\
(-0.005,0.001)\end{array}$ & $\begin{array}{l}\uparrow: 0.000 \\
(-0.002 \\
0.001)\end{array}$ & $\begin{array}{l}\downarrow:-0.000 \\
(-0.002 \\
0.009)\end{array}$ & $\begin{array}{l}\downarrow:-0.001 \\
(-0.003 \\
0.002)\end{array}$ & $\begin{array}{l}\uparrow: 0.002 \\
(0.000 \\
0.003)^{*}\end{array}$ & $\begin{array}{l}\downarrow:-0.000 \\
(-0.001 \\
0.000)\end{array}$ & $\begin{array}{l}\downarrow:-0.005 \\
(-0.022 \\
0.011)\end{array}$ \\
\hline \multirow[t]{4}{*}{ [46] } & \multirow{4}{*}{$\begin{array}{l}\text { Yang } \\
\text { China } \\
2016\end{array}$} & \multirow{4}{*}{$\begin{array}{l}\text { Spearmans } \\
\text { partial } \\
\text { correlation } \\
\text { analysis } \\
\text { Maternal exposure used. }\end{array}$} & FT3 & $\uparrow: 0.124$ & $\uparrow: 0.025$ & $\uparrow: 0.024$ & $\downarrow:-0.063$ & $\downarrow:-0.087$ & $\downarrow:-0.121$ & $\downarrow:-0.268^{*}$ \\
\hline & & & FT4 & $\uparrow: 0.038$ & $\downarrow:-0.057$ & $\uparrow: 0.000$ & $\downarrow:-0.072$ & $\downarrow:-0.086$ & $\downarrow:-0.062$ & $\downarrow:-0.160^{*}$ \\
\hline & & & $\pi 3$ & $\uparrow: 0.084$ & $\uparrow: 0.008$ & $\uparrow: 0.102$ & $\downarrow:-0.018$ & $\downarrow:-0.079$ & $\downarrow:-0.097$ & $\downarrow:-0.301^{*}$ \\
\hline & & & $\pi 4$ & $\uparrow: 0.084$ & $\uparrow: 0.021$ & $\uparrow: 0.062$ & $\downarrow:-0.006$ & $\downarrow:-0.010$ & $\uparrow: 0.030$ & $\downarrow:-0.160^{*}$ \\
\hline \multirow[t]{5}{*}{ [47] } & \multirow{5}{*}{$\begin{array}{l}\text { Xiao } \\
\text { Faroe } \\
\text { Island } \\
2019\end{array}$} & \multirow{5}{*}{$\begin{array}{l}\text { Percent change in thyroid } \\
\text { hormone levels per doubling } \\
\text { of PFAA concentrations ( } 95 \% \text { Cl) }\end{array}$} & FT4I & $\begin{array}{l}\uparrow: 4.4 \\
(-2.3,11.6)\end{array}$ & $\begin{array}{l}\downarrow:-0.8 \\
(-8.2,7.4)\end{array}$ & $\begin{array}{l}\downarrow:-0.6 \\
(-6.1,5.1)\end{array}$ & $\begin{array}{l}\downarrow:-1.2 \\
(-8.9,7.3)\end{array}$ & $\begin{array}{l}\uparrow: 0.2 \\
(-7.2,8.1)\end{array}$ & $\begin{array}{l}\uparrow: 2.3 \\
(-3.1,8.1)\end{array}$ & $\begin{array}{l}\uparrow: 0.2 \\
(-2.2,2.6)\end{array}$ \\
\hline & & & FT3 & $\begin{array}{l}\downarrow:-0.7 \\
(-5.6,4.5)\end{array}$ & $\begin{array}{l}\uparrow: 2.2 \\
(-3.6,8.5)\end{array}$ & $\begin{array}{l}\uparrow: 3.1 \\
(-1.2,7.6)\end{array}$ & $\begin{array}{l}\downarrow:-2.7 \\
(-8.5,3.6)\end{array}$ & $\begin{array}{l}\downarrow:-1.4 \\
(-6.9,4.5)\end{array}$ & $\begin{array}{l}\downarrow:-1.8 \\
(-5.8,2.4)\end{array}$ & $\begin{array}{l}\uparrow: 0.6 \\
(-1.3,2.4)\end{array}$ \\
\hline & & & FT4 & $\begin{array}{l}\uparrow: 5.9 \\
(-0.2,12.4)\end{array}$ & $\begin{array}{l}\downarrow:-2.9 \\
(-9.4,4.3)\end{array}$ & $\begin{array}{l}\downarrow:-0.4 \\
(-5.4,4.8)\end{array}$ & $\begin{array}{l}\downarrow:-5.7 \\
(-12.4,1.4)\end{array}$ & $\begin{array}{l}\downarrow:-1.5 \\
(-8.1,5.5)\end{array}$ & $\begin{array}{l}\downarrow:-0.1 \\
(-5.0,5.0)\end{array}$ & $\begin{array}{l}\uparrow: 0.2 \\
(-2.0,2.4)\end{array}$ \\
\hline & & & $\Pi 4$ & $\begin{array}{l}\uparrow: 0.4 \\
(-6.9,8.2)\end{array}$ & $\begin{array}{l}\uparrow: 0.1 \\
(-8.3,9.3)\end{array}$ & $\begin{array}{l}\uparrow: 0.7 \\
(-5.5,7.3)\end{array}$ & $\begin{array}{l}\downarrow:-1.2 \\
(-9.9,8.2)\end{array}$ & $\begin{array}{l}\downarrow:-2.1 \\
(-10.1,6.7)\end{array}$ & $\begin{array}{l}\uparrow: 0.4 \\
(-5.6,6.9)\end{array}$ & $\begin{array}{l}\uparrow: 0.1 \\
(-2.6,2.9)\end{array}$ \\
\hline & & & T3RU & $\begin{array}{l}\uparrow: 4.0 \\
(-0.4,8.5)\end{array}$ & $\begin{array}{l}\downarrow:-0.9 \\
(-5.8,4.3)\end{array}$ & $\begin{array}{l}\downarrow:-1.3 \\
(-4.9,2.3)\end{array}$ & $\begin{array}{l}\uparrow: 0.1 \\
(-5.1,5.5)\end{array}$ & $\begin{array}{l}\uparrow: 2.2 \\
(-2.7,7.5)\end{array}$ & $\begin{array}{l}\uparrow: 1.9 \\
(-1.7,5.7)\end{array}$ & $\begin{array}{l}\uparrow: 0.1 \\
(-1.5,1.7)\end{array}$ \\
\hline
\end{tabular}


Table 8 Association of maternal PFAAs exposure and maternal T4 and T3 by trimester (adjusted results) (Continued)

\begin{tabular}{|c|c|c|c|c|c|c|c|c|c|c|}
\hline Reference & $\begin{array}{l}\text { Author } \\
\text { Country } \\
\text { Year } \\
\end{array}$ & Effect estimate & $\mathrm{TH}$ & PFHxS & PFOS & PFOA & PFNA & PFDA & PFUnA & PFDoA \\
\hline \multicolumn{11}{|c|}{ TH measured during multiple timepoints } \\
\hline \multirow[t]{8}{*}{ [43] } & \multirow{8}{*}{$\begin{array}{l}\text { Berg } \\
\text { Norway } \\
2015\end{array}$} & \multirow{8}{*}{$\begin{array}{l}\text { Mixed effects model estimated } \\
\text { mean differences in thyroid } \\
\text { hormone concentrations over } \\
\text { time (2nd trimester, at birth and } \\
6 \text { weeks postpartum). Change } \\
\text { across exposure quartiles } \\
\text { (Q1 reference). NS: results } \\
\text { not stated. }\end{array}$} & \multirow[t]{3}{*}{$\pi 3$} & \multirow[t]{3}{*}{ NS } & \multirow[t]{3}{*}{ NS } & \multirow[t]{3}{*}{ NS } & \multirow[t]{3}{*}{ NS } & $\begin{array}{l}\downarrow: \text { Q2: }-0.04 \\
(-0.08 .0 .04)\end{array}$ & \multirow[t]{3}{*}{ NS } & \\
\hline & & & & & & & & $\begin{array}{l}\downarrow: \text { Q3: }-0.05 \\
(-0.08,0.00)\end{array}$ & & - \\
\hline & & & & & & & & $\begin{array}{l}\downarrow: Q 4:-0.1 \\
(-0.14 \\
-0.06)^{*}\end{array}$ & & \\
\hline & & & \multirow[t]{3}{*}{ FT3 } & \multirow[t]{3}{*}{ NS } & \multirow[t]{3}{*}{ NS } & \multirow[t]{3}{*}{ NS } & \multirow[t]{3}{*}{ NS } & \multirow[t]{3}{*}{ NS } & $\begin{array}{l}\downarrow \text { Q2: }-0.08 \\
(-0.15,-0.00)^{*}\end{array}$ & \\
\hline & & & & & & & & & $\begin{array}{l}\downarrow: \text { Q3: }-0.09 \\
(-0.16,-0.01)^{*}\end{array}$ & - \\
\hline & & & & & & & & & $\begin{array}{l}\downarrow \text { Q4: }-0.18 \\
(-0.25, \\
-0.12)^{*}\end{array}$ & \\
\hline & & & $\pi 4$ & NS & NS & NS & NS & NS & NS & - \\
\hline & & & FT4 & NS & NS & NS & NS & NS & NS & - \\
\hline \multirow[t]{2}{*}{ [45] } & \multirow{2}{*}{$\begin{array}{l}\text { Reardon } \\
\text { Canada } \\
2019\end{array}$} & \multirow{2}{*}{$\begin{array}{l}\text { Overall main effect of PFAAs on } \\
\text { TH (all timepoints) from mixed } \\
\text { effects models: Adjusted } \beta \\
(p \text {-value) }\end{array}$} & FT4 & $\begin{array}{l}\downarrow:-0.006 \\
(0.034)^{*}\end{array}$ & $\begin{array}{l}\downarrow:-0.000 \\
(0.999)\end{array}$ & $\begin{array}{l}\downarrow:-0.002 \\
(0.138)\end{array}$ & $\begin{array}{l}\downarrow:-0.004 \\
(0.379)\end{array}$ & $\begin{array}{l}\downarrow:-0.010 \\
(0.314)\end{array}$ & $\begin{array}{l}\downarrow:-0.037 \\
(0.321)\end{array}$ & - \\
\hline & & & FT3 & $\uparrow: 0.000(0.955)$ & $\begin{array}{l}\uparrow:-0.003 \\
(0.242)\end{array}$ & $\begin{array}{l}\uparrow: 0.001 \\
(0.535)\end{array}$ & $\begin{array}{l}\uparrow: 0.004 \\
(0.330)\end{array}$ & $\begin{array}{l}\uparrow: 0.003 \\
(0.736)\end{array}$ & $\begin{array}{l}\uparrow: 0.014 \\
(0.698)\end{array}$ & - \\
\hline
\end{tabular}

TT3 (total triiodothyronine), FT3 (total triiodothyronine), TT4 (total thyroxine), FT4 (free thyroxine), FT4l (free thyroxine index), $\downarrow$ decreasing, $\uparrow$ increasing, bold format and star* indicate significant results $P<0.05$, NS: results not stated, -: PFAAs not examined, Q1 (1st quartile), Q2 (2nd quartile), Q3 (3rd quartile), Q4 (4th quartile), IQR (interquartile range)

PFAAs include: PFOS Perfluorooctane sulfonate, PFOA Perfluorooctanoate, PFHXS Perfluorohexane sulfonate, PFNA Perfluorononanoic acid, PFDA Perfluorodecanoic acid, PFUnA Perfluoroundecanoic acid, PFDoA Perfluorododecanoic acid

[44] or at multiple time points [45]. Two out of ten studies found an inverse association between PFOS and maternal TSH levels (in the 1st [38] and 3rd trimester [46]). However, two other studies found PFOS significantly positively associated with maternal TSH levels at the 2nd trimester [41] and at multiple timepoints [43], respectively. In fact, women with the highest PFOS concentration had a significant $24 \%$ higher TSH levels than those with the lowest PFOS concentration at multiple time points (data not shown) [43].

No significant associations were found between maternal PFOA concentration and TSH levels (Table 7).

Among nine studies, one study found a positive association between PFNA concentration and maternal TSH levels (Table 7) in the 2nd trimester [42], while another study found an inverse association within 3rd trimester TSH [46].

One out of seven studies found a significant inverse association between PFDA and maternal 3rd trimester TSH levels (Table 7) [46].

The association between maternal PFUnA and TSH was examined in six studies (Table 7), and Yang et al. 2016 [46] observed a significantly inverse association in the 3rd trimester. In the same study [46] the maternal PFDoA concentration and TSH level were as well found inversely associated in the 3rd trimester (Table 7).

\section{Maternal PFAAs exposure and T4 and T3 by trimester (Table 8)}

Maternal T4 and T3 were assessed by nine studies (Table 8). Significantly inverse associations were observed between maternal FT4 effect estimates (FT4I) and PFHxS levels in the 1st trimester [39] and at multiple time points [45]. Although positive tendencies were found, none of the results were significant for PFOS exposure and maternal T4 and T3 levels (Table 8). Out of nine studies, investigating the association maternal PFOA concentration with T4 and T3 levels (Table 8), one study found a significantly inverse association with maternal 1st trimester FT4I levels when comparing the 2nd and 4th quartiles with the lowest exposure quartile [39]. Among the eight studies evaluating maternal PFNA concentrations and T4 and T3 levels (Table 8), one study found that PFNA concentrations inversely associated with maternal FT4 and TT4 levels at the 3rd trimester [44]. Among six studies (Table 8), one study reported a positive association of PFDA and maternal TT3 in the 3rd trimester [44], while another study observed a significantly negative association of high PFDA concentration (4th quartile) and TT3 at multiple time 


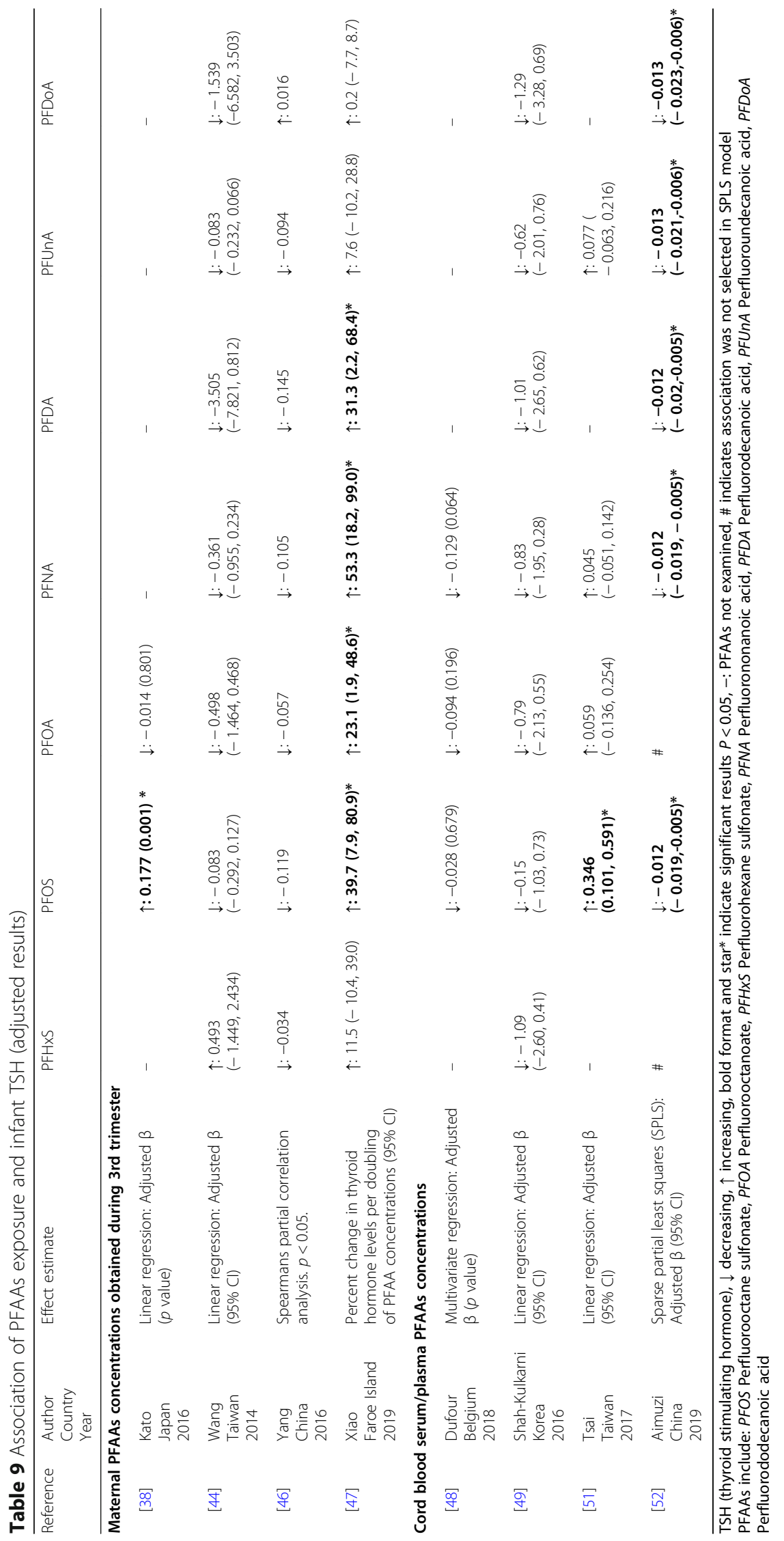




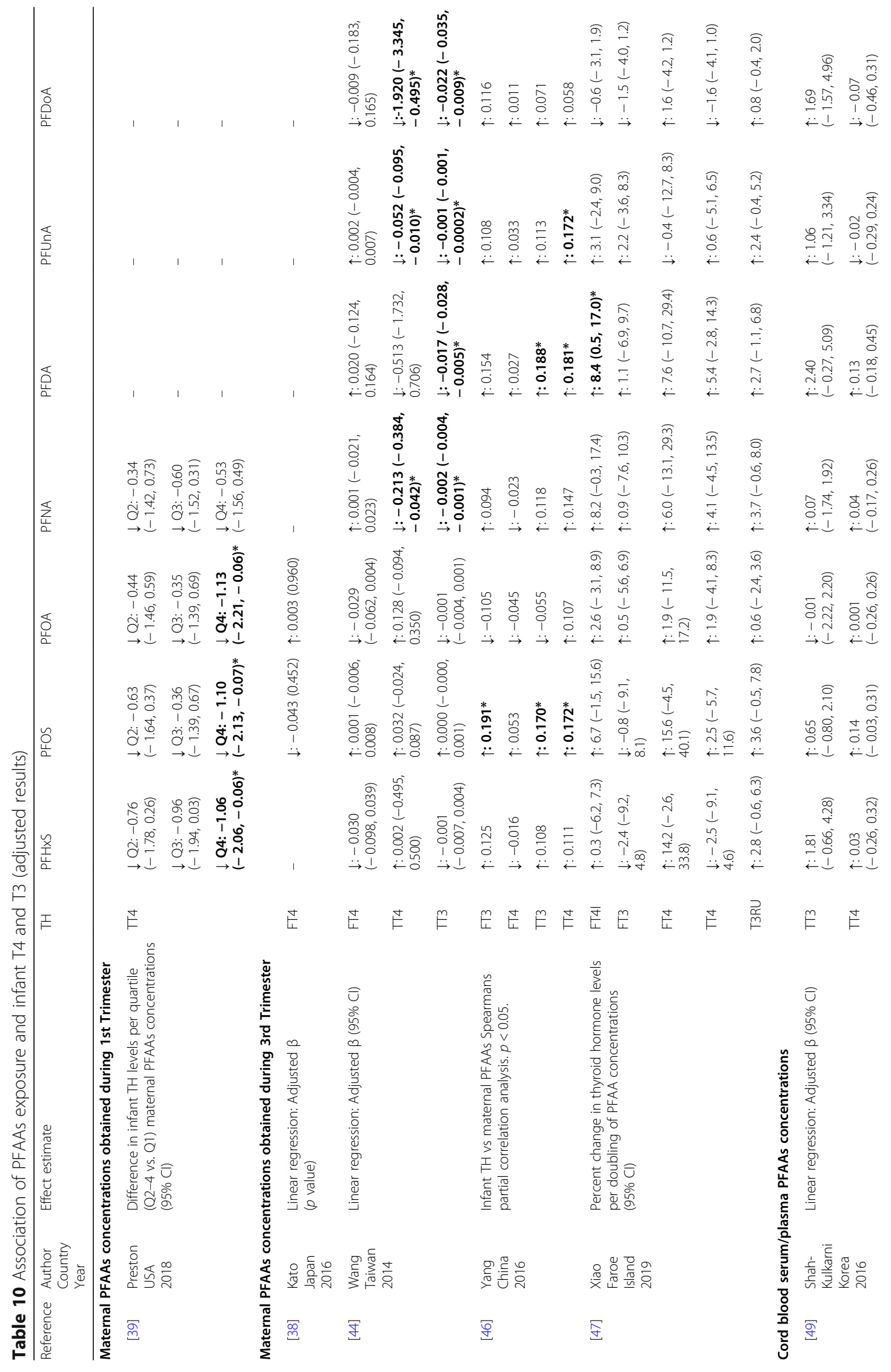




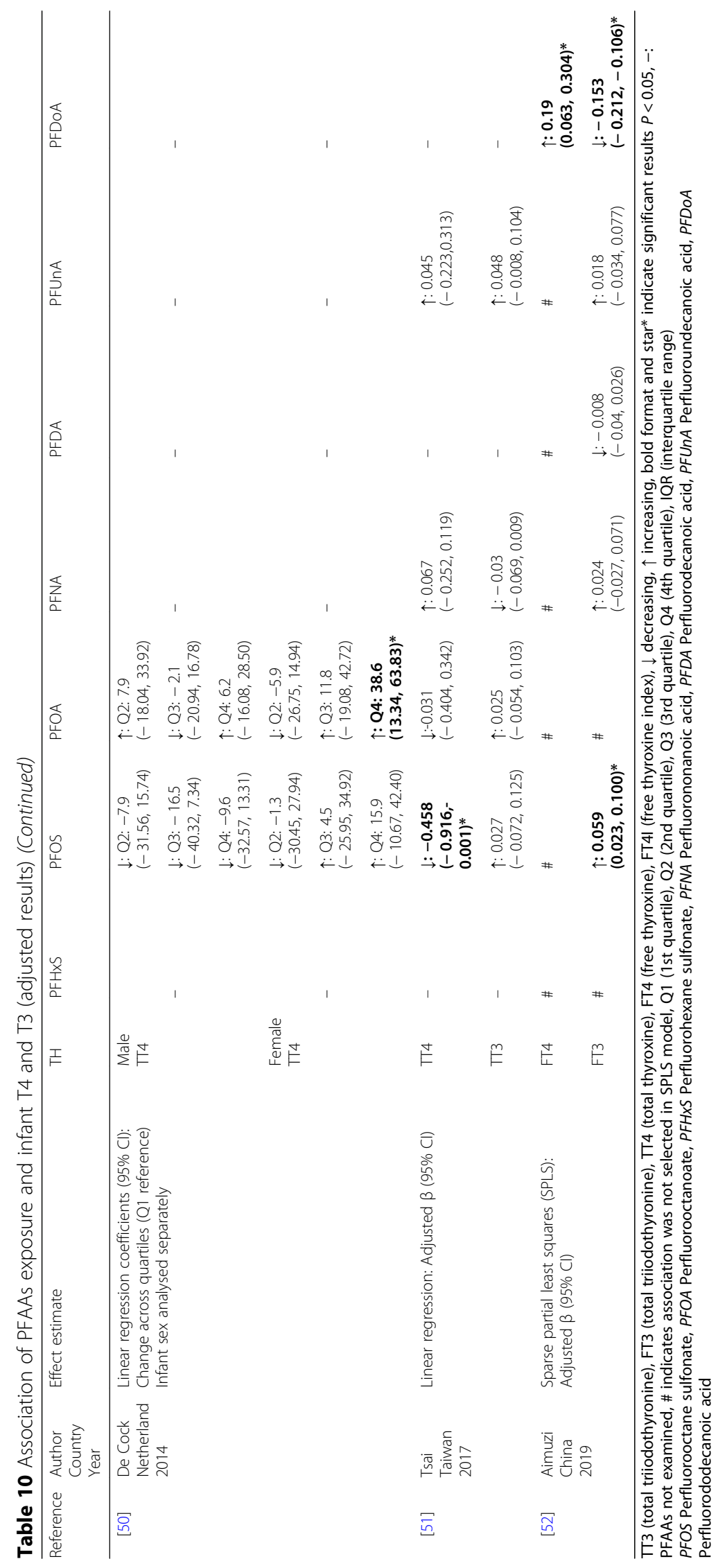


points [43]. Out of five studies assessing maternal $\mathrm{T} 4$ and T3 (Table 8), only one showed that FT3 measured at multiple time points decreased with high PFUnA concentrations (4th quartile) [43], while another study found inverse association between FT4 and TT4 and PFUnA exposure in the 3rd trimester [44]. Maternal T4 and T3 versus PFDoA exposure were assessed by three studies (Table 8); two studies observed a significantly inverse association with maternal 3rd trimester FT3, TT3, FT4 and TT4 levels [44, 46].

\section{PFAAs exposure and infant TSH level (Table 9)}

PFHxS exposure elicited no significant association and no clear trend after examining four studies with infant TSH data (Table 9).

Evaluating the PFOS exposure data in the 3rd trimester or cord blood, the infant TSH levels were examined in eight studies (Table 9). Maternal 3rd trimester [38, 47] and cord blood PFOS concentrations [51] were significantly positively associated with infant TSH levels (Table 9). But, an inverse association was found in another study assessing PFOS in cord blood and infant TSH [52]. Upon stratification by infant sex, three studies found positive associations among boys [38]: $\beta_{\text {male }}=$ 0.205, $p=0.014$, [47]: \% change per doubling in PFOS $=$ $39.5,95 \%$ CI $(0.4,94.1)[51]: \beta_{\text {male }}=0.333,95 \%$ CI $(0.012$, 0.678)] (Suppl. Table 1a). While among female infants, one study found positive [38] $\left[\beta_{\text {female }}=0.173, p=0.021\right.$ ] and one inverse [52] $\left[\beta_{\text {female }}=-0.016,95 \%\right.$ CI $(-0.032$, - 0.002)] association, respectively (Suppl. Table 1b).

Using cord blood or maternal 3rd trimester PFOA exposure, the association of PFOA with infant TSH was examined in eight studies (Table 9). A positively significant association was found in one study with maternal 3rd trimester PFOA concentrations [47], while negative non-significant tendencies were seen in several other studies [38, 44, 46, 48, 49].

Infant TSH was assessed upon PFNA exposure in seven studies (Table 9), and a positive association was seen with maternal PFNA concentrations at the 3rd trimester [47], while an inverse association was found with cord blood PFNA concentrations [52]. When stratifying by infant sex (Suppl. Table 1a and b), for males, a significantly positive association with maternal 3rd trimester PFNA concentrations [\% change per doubling in PFNAmale $=60.6,95 \% \mathrm{CI}(15.0,124.6)][47]$ and an inverse association with cord blood PFNA concentrations $\left[\beta_{\text {male }}=-\right.$ 0.166, $p=0.018$ ] [48] was found. Among girls, a similar pattern for TSH was seen with a positive association with maternal 3rd trimester PFNA concentrations [\% change per doubling in $\mathrm{PFNA}_{\text {female }}=46.7,95 \% \mathrm{CI}$ (5.9, 103.2)] [47] and an inverse association with cord blood PFNA concentrations $\left[\beta_{\text {female }}=-1.69,95 \%\right.$ CI $(-3.31$, 0.08)] [49].
Infant TSH (Table 9) was found positively associated with maternal 3rd trimester PFDA concentrations [47], while an inverse association was found with cord blood PFDA concentrations [52]. Other studies also showed decreasing tendencies (in maternal and cord blood), however the associations were non-significant [44, 46, 49]. When stratifying by infant sex (Suppl. Table 1a and b), infant TSH was positive associated with maternal PFDA in 3rd trimester concentrations among boys [\% change per doubling in $\mathrm{PFDA}_{\text {male }}=37.0,95 \% \mathrm{CI}(0.5$, 86.8)] [47].

The relation between infant TSH level and PFUnA concentration were examined in six studies (Table 9) and one study showed a significantly inverse association with cord blood PFUnA concentrations [52].

Among five studies that examined cord blood PFDoA concentration and infant TSH level (Table 9), one study found a significantly inverse association [52]. After stratification by infant sex, the inverse association was only significant among boys [52] $\left[\beta_{\text {male }}=-0.062,95 \% \mathrm{CI}\right.$ $(-0.108 ;-0.017)]$ (Suppl. Table 1a).

\section{PFAAs exposure and infant T4 and T3 levels (Table 10)}

PFHxS exposure and infant T4 and T3 levels were evaluated by six studies (Table 10) and a study found high maternal PFHxS levels (4th quartile) were significantly inversely associated with infant TT4 levels [39]. After stratification by infant sex, this TT4 association remained significant only among males $\left[\mathrm{Q} 4: \beta_{\text {male }}=-\right.$ 2.51, 95\%CI $(-3.99,-1.04)$ ] [39] (Suppl. Table 2a). Whereas, cord blood PFHxS levels were associated with increased female infant TT3 levels $\left[\beta_{\text {female }}=4.28,95 \% \mathrm{CI}\right.$ $(0.39,8.17)]$ [49] (Suppl. Table 2b).

The assessement of PFOS exposure and infant T4 and T3 found in four studies significant associations (Table 10). High PFOS concentration in the 1st trimester (4th quartile) [39] and in cord blood [51] was inversely associated with infant TT4 levels (Table 10). In contrast, maternal 3rd trimester PFOS concentration [46] was significantly and positively associated with increased infant TT3, FT3 and TT4 levels. Likewise, infant cord blood PFOS concentrations [52] was positively associated with FT3 levels (Table 10). Upon stratification by infant sex, the significant inverse associations with TT4 remained only among boys ([Q4 $\beta_{\text {male }}=-2.20$, $95 \% \mathrm{CI}(-3.74 ;-0.66)]$ and $\left[\beta_{\text {male }}=-0.667,95 \% \mathrm{CI}(-\right.$ $1.28 ;-0.05)]$ ) $[39,51]$ (Suppl. Table 2a), while one study found a positive association with FT4I among girls [\% change per doubling in PFOS $_{\text {female }}=13.2,95 \%$ CI $(0.9$, 27.1)] [47] (Suppl. Table 2b).

One study, out of nine, found infant TT4 levels (Table 10) to be significantly and inversely associated with higher maternal PFOA concentration (4th quartile) in the 1st trimester [39]. After stratification by sex, the significance for TT4 
remained only among boys $\left[\mathrm{Q} 4 \beta_{\text {male }}=-1.72,95 \% \mathrm{CI}(-\right.$ 3.36; - 0.08)] [39] (Suppl. Table 2a). Another study found that cord blood PFOA concentration was positively associated with FT4 in males [52] $\left[\beta_{\text {male }}=0.062,95 \% \mathrm{CI}(0.024\right.$; 0.138)] (Suppl. Table 2a). Whereas, increased TT4 levels were found in girls with the highest cord blood PFOA concentration (4th quartile) $\left[\mathrm{Q} 4 \beta_{\text {female }}=38.6,95 \% \mathrm{CI}\right.$ (13.34; 63.83)] [50] (Table 10, Suppl. Table 2b).

Infant T4 and T3 versus PFNA exposure was investigated in seven studies (Table 10) and one study found that infant TT3 and TT4 were negatively associated with maternal PFNA concentrations at 3rd trimester [44]. After stratification by sex, FT4 was found positively associated with cord blood PFNA concentration in boys [52] [ $\beta_{\text {male }}=$ 0.04, 95\%CI (0.006, 0.081)] (Suppl. Table 2a).

Five studies investigated the infant $\mathrm{T} 4$ and $\mathrm{T} 3$ levels versus PFDA exposure (Table 10). Wang et al. 2014 demonstrated that maternal 3rd trimester PFDA concentration was inversely [44] and Yang et al. 2016 found a positively [46] association with infant TT3 levels. Likewise, infant TT4 and FT4I were positively associated with maternal 3rd trimester PFDA concentrations [46, 47]. After stratification by infant sex, FT4 was found positively associated with cord blood PFDA concentrations for males [52] $\left[\beta_{\text {male }}=0.043,95 \% \mathrm{CI}(0.016 ; 0.078)\right]$. Moreover, a positive association between PFDA and FT4I in males was observed [\% change per doubling in $\mathrm{PFDA}_{\text {male }}=11.5,95 \% \mathrm{CI}$ $(1.5 ; 22.6)]$ [47] (Suppl. Table 2a).

In two out of six studies significant data was found for exposure to PFUnA and association to infant T4 and T3 levels (Table 10). Maternal 3rd trimester PFUnA concentration was associated inversely with TT3 and TT4 infant levels in one study [44], and another study found increasing infant TT4 levels [46]. Tsai et al. found no effect in the total study population but a significant positive association when examining the 60th-89th percentile of cord blood PFUnA concentration with infant T3 levels $[\beta=0.197$, 95\% CI (0.031;0.364), $P<0.05$ ] (data not shown) [51].

For PFDoA exposure versus infant T4 and T3 levels two of five studies found significant associations (Table 10). An inverse association with maternal 3rd trimester PFDoA was found in one study [44]. Cord blood PFDoA concentration was found negatively associated with infant FT3 but positively with FT4 [52]. After stratification by infant sex, FT4 was positively associated with cord blood PFDoA concentration in both boys and girls $\left(\left[\beta_{\text {male }}(\mathrm{FT} 4)=0.54,95 \% \mathrm{CI}\right.\right.$ $(0.019 ; 0.119)]$, $\left[\beta_{\text {female }}(\mathrm{FT} 4)=0.174,95 \% \mathrm{CI}(0.019 ; 0.331)\right]$ [52], but for FT3, the inverse significant association remained only among girls $\left[\beta_{\text {female }}(\mathrm{FT} 3)=-0.124,95 \% \mathrm{CI}\right.$ $(-0.185 ;-0.056)]$ [52] (Suppl. Table 2a and b).

\section{Discussion}

This literature review suggests that PFAAs exposure can influence maternal TSH levels. Moreover, several PFAAs were inversely associated with maternal $\mathrm{T} 4$ and $\mathrm{T} 3$ levels. The studies reported some associations between PFAAs exposure and infant $\mathrm{TH}$ levels although the data was less conclusive.

\section{TSH}

PFHxS, PFOS, PFNA, PFDA, PFUnA and PFDoA exposure seem to affect maternal TSH levels; however, only PFHxS, PFOS and PFNA were found significantly associated with TSH in more than one of the reviewed studies. Thus, five studies found significant increased maternal TSH levels upon exposure to PFOS, PFNA and PFHxS [41-45], which might indicate a (subclinical) hypothyroid maternal state. Two studies found significantly decreased maternal TSH levels with increasing PFOS, PFNA, PFDA, PFUnA and PFDoA concentrations [38, 46], which might indicate a hyperthyroid maternal state. None of the ten studies investigating the association between PFOA concentration and maternal TSH found any significant results [38-47]. However, it should be noted that all these PFAAs are often highly intercorrelated.

Three studies found increased TSH levels in infant upon PFOS exposure [38, 47,51], while one study also found increased infant TSH levels with PFOA, PFNA and PFDA concentrations [47]. A single study found decreasing infant TSH with PFOS, PFNA, PFDA, PFUnA and PFDoA concentrations [52]. None of the studies investigating the association between $\mathrm{PFHxS}(n=5)$ and infant TSH found any significant associations [44, 46, $47,49,52$ ] and seven of the eight studies investigating the association with PFOA found non-significant results $[38,44,46,48,49,51,52]$.

\section{T4 and T3}

Five studies found decreasing maternal TT3, TT4, FT3, FT4 and FT4I levels associated with PFHxS, PFOA, PFNA, PFDA, PFUnA and PFDoA concentrations [39, 43-46]. Low levels of T4 and T3 (and high TSH) could indicate a hypothyroid maternal state. One study showed a positive association of maternal TT3 upon PFDA exposure [44].

Four studies found decreasing infant TT4, TT3 and FT3 with exposure to any of the investigated PFAAs [39, $44,51,52]$, and four studies found increasing infant FT3, FT4, FT4I, TT3 and TT4 levels with exposure to PFOS, PFOA, PFDA, PFUnA and PFDoA [46, 47, 50, 52].

In overall, most studies showed a positive association of maternal TSH and a possible negative association of maternal T4 and T3 upon PFAAs exposure. This indicates that pregnant women may be at risk of developing a hypothyroid state with increasing PFAAs exposure. Infant $\mathrm{TH}$ might also be affected, mainly increasing infant TSH levels with PFOS. More studies are needed to 
determine the direction of the association with infant $\mathrm{T} 4$ and T3 levels.

\section{Evaluation of TH across pregnancy trimesters with PFOS and PFOA exposure}

During the 1st trimester, most studies on maternal $\mathrm{TH}$ $[38,39]$ (Table 7) showed that TSH was inversely related with PFOS, although, only one reached statistical significance [38]. Non-significant positive relationships were found with PFOA concentrations. PFOS and PFOA concentrations was mainly non-significantly but positively associated to maternal FT4 levels $[38,40]$ and TT4 levels [39] (Table 8). FT4I was inversely associated to PFOA but not significantly to PFOS [39].

In the 2nd trimester, studies on maternal TH $[41,42]$ (Table 7) showed that TSH was positively associated with PFOS exposure, being statistically significant in one study [41]. No consistency was found for the relation between PFOA and TSH, with both positive and inverse non-significant associations [41, 42]. Moreover, results on maternal T4 (Table 8) did not show any significant associations [42].

In the 3rd trimester, studies on maternal $\mathrm{TH}[44,46$, 47] (Table 7) demonstrated that TSH was significantly and inversely associated with maternal PFOS concentration in one study [46]. No consistent direction was found for PFOA exposure. For the maternal T4 and T3 levels [44, 46, 47] (Table 8), FT4, TT4, FT3 and TT3 were mainly positively, but non-significantly, associated with PFOS and PFOA.

Two studies conducted a longitudinal assessment of $\mathrm{TH}$ measurements across the trimesters and postpartum (Tables 2, 7 and 8) [43, 45]. Repeated measurements of maternal TSH were positively associated with PFOS exposure being significant in one study [43] (Table 7). A non-significantly inverse association was found for FT4, but positive for FT3 upon exposure to PFOS and PFOA [45](Table 8).

The trimester data evaluation showed negative associations between PFOS and maternal TSH in the 1st and 3rd trimester, but positive during the 2nd trimester (Table 7), whereas PFOA exposure did not elicite significant association to TSH in any of the trimesters. The trimester data evaluations for maternal T4 and T3 versus PFOS and PFOA concentrations showed inverse association between PFOA and FT4I in first trimester [39] (Table 8). Longitudinal TH studies found a positive association between TSH and PFOS [43] while data on PFOA exposure did not imply a strong association with maternal thyroid hormones. It must be noted that only few studies with information on each trimester were found, thus, the direction of the estimates might change when more studies are performed. Interestingly, it has been shown that associations of maternal TSH with exposure to PFHxS and branched PFOS isomers were strongest in early pregnancy (1st trimester) and weakened over subsequent trimesters (data not shown) [45].

\section{Stratifying by infant sex}

Seven studies stratified on infant sex [38, 39, 47-49, 51, 52] (Suppl. Tables 1 and 2).

PFOS concentration was positively and significantly associated with TSH levels in boys $[38,47,51]$ and girls [38]. PFNA was found to be inversely significantly associated with TSH levels in boys [48] and girls [49], while another study found positive associations in both genders [47] (Suppl. Table 1a-b). One study observed a negative association of TSH with cord blood PFOS exposure in girls and PFDoA in boys (Suppl. Table 1a-b) but they did not perform the statistical analysis on all PFAAs congeners in each sex due to their choice of statistical model (sparse partial least squares) [52].

In boys, TT4 levels were inversely associated with three PFAAs congeners (PFHxS, PFOS and PFOA) measured in maternal blood and cord blood samples (Suppl. Table 2a) [39, 51] and PNFA maternal concentrations were positively associated with FT4I [47]. In girls, TT3 levels were positively associated with cord blood PFHxS [49], and FT4I and TT4 levels positively associated with maternal PFOS and PFOA and cord blood PFOA (highest quartile) $[47,50]$ (Suppl. Table $2 b$ ). In boys, a study found significant positive associations for FT4 with several cord blood PFAAs (PFOA, PFNA, PFDA, PFDoA) concentration (Suppl.Table 2a), the analysis was however not performed in girls due to their choice of statistical model (sparse partial least squares) [52]. In the pooled infant analyses (Table 10) this study observed similar significant positive association between FT4 level, but inversely association with FT3 level, and cord blood PFDoA concentration [52].

The reviewed studies do not allow us to establish a distinct tendency or greater sensitivity regarding infant sex. Further studies must be conducted to state any conclusion on gender specific sensitivity for PFAAs exposure and $\mathrm{TH}$ disruption.

\section{Previous review literature}

The possible link between PFAA exposure and thyroid outcomes in pregnant women was previously reviewed by Ballesteros et al. in 2017 [53]. They included 10 epidemiological studies on PFOS, PFOA, PFHxS and PFNA exposure during prenatal life, childhood and adolescence (up to 19 years), with sample size ranging from 40 to > 10.000 participants. In accordance to this current review, Ballesteros et al. suggested a positive association between PFHxS and PFOS and maternal TSH levels, as well as, between PFNA and TSH levels measured in boys aged > 11 years. Compared to Ballesteros et al. [53], the current 
review includes recent epidemiological studies (enrolment year: 1996-2016 vs. 1987-2013), more studies ( $n=15$ vs. $n=10$ ), bigger sample size (participants $>100$ vs. $>=43$ ) and covers more PFAAs congeners (7 vs. 4 ), thus it might add a stronger study power and support to the previous observations. We also found stronger evidence of a positive relationship between PFAAs exposure (especially PFOS) with maternal TSH, and a possible inverse relationship with maternal T4 and T3 levels.

\section{Biological plausibility}

PFAAs are endocrine disrupting compounds that impact on endogenous hormone homeostasis [54]. Different pathways have been suggested to explain thyroid disrupting effects: PFAAs may induce increased excretion of T4 by alteration of TBG due to competitive binding [55], and/or increased conversion of T4 to T3 by type 1 de-ionidase in hepatic cells, as well as increased hepatic metabolism of T4 [56]. PFAAs could furthermore alter the responsiveness of the HPT axis [57], as well as, alter the HPG homeostasis and their signaling pathways which might affect foetal growth $[58,59]$.

Several animal studies have shown reduced foetal growth upon PFAAs exposure [60-62]. Furthermore, animal studies have reported PFAAs disrupted HPT [60, 63, 64] and HPG-axis [65, 66]. However, comparison among studies is challenged because interspecies diversity (e.g. half-lives, excretion and plasmatic carrier proteins), PFAA congeners and levels of exposure, and extrapolation from animal studies to human risk assessment $[64,67]$.

Studies have suggested that thyroid and oestrogenic/ androgenic pathways may be a part conjoined mechanisms. In vitro studies have shown that the HPT axis is affected by PFAAs exposure by inhibiting thyroid receptors, antagonizing thyroid cell proliferation [57]. Furthermore, PFOS and PFOA decreased TPO activity, blocking the ionizing process of thyroglobin [37], leading to reduced T4 and T3 levels. Cell proliferation of the THdependent rat pituitary GH3 cells was shown to be dependent on the involvement of the oestrogen receptor [68]. Moreover, in vitro studies have also shown alterations in the biosynthesis of oestrogens and interference with the oestrogen receptor, after exposure of PFNA, PFDA, PFHxS, PFOS and PFOA, due to their oestrogenic and anti-androgenic potential [2, 69-71]. Extracted PFAA mixtures from human serum samples also activated oestrogenic receptors $[72,73]$ and in an epidemiological study serum PFAAs mixture induced estrogenic activity in pregnant women was associated with significantly lower birth weight and birth length [58]. Most studies examined the effect of single PFAA congeners. More studies are needed for the combined cocktail effect of PFAAs mixtures and/or other POPs, which may have additively and/or synergistic actions $[58,73]$.

\section{PFAAs exposure and TH outcome assessment}

All the reviewed studies determined PFAA concentrations, using small variations of the same methodology (liquid chromatography-tandem mass spectrometry), in mother and/or infant. Maternal biological matrix was either blood plasma or serum and for infant concentrations either cord blood plasma or serum. The LOD and LOQ values were different among studies. The percentages of samples below LOD/LOQ varied among studies, especially for PFHxS, PFNA, PFDA in maternal serum/ plasma samples (Table 3) and for most PFAA congeners in cord blood (Table 4). Nevertheless, most selected studies handled the PFAA concentrations below LOD/ LOQ with replacement by LOD/LOQ divided by the square root of 2 . The sensitivity of the used methods might influence the estimated effect but the exposure levels is expected to be the main factor for the exposure - outcome associations.

Umbilical cord blood samples were also used to determine both infant exposure and thyroid function having the benefit being of representing the fetal environment. However, there are also some limitations of using cord blood for infant thyroid function assessment. Firstly, cord blood thyroid hormone levels likely reflects a combination of maternal and fetal hyroid hormones. Secondly, maternal and umbilical cord blood PFAAs concentrations are highly correlated $[74,75]$, and the ability of PFAAs to cross the placenta differs between compounds (i.e. PFOA passes more easily than PFOS $[20,75,76]$.

Maternal PFAA concentrations might not be constant throughout pregnancy due to physiological hemodynamic changes. PFAAs concentrations decrease during pregnancy due to increased plasma volumes [77] and higher renal PFAAs excretion [78]. Thus, measurement of PFAAs in late pregnancy might not be directly compared with PFAAs concentration in 1st trimester [77]. Moreover, women are able to transfer some of their PFAAs burden to their offspring by placental $[21,79]$ and post-partum breast feeding [80]. This complicates comparison between studies with different pregnancy time points of exposure assessment [19]. Even more, one study measured the thyroid hormone outcome before exposure (PFAAs) [38], which could cause concern of causality, although some retrospective consistency for PFAAs exposure can be assumed due to their persistent nature (long half-lives in human serum) $[15,18]$. However, a study showed the PFAA concentrations measured in the 1st and 3rd trimester were highly correlated $(r>0.64)$ [81] and argued that a single exposure measurement might be considered as a representative of serum levels during pregnancy.

In most of the studies, the exposure was evaluated as single congeners and not as the real mixture/combined exposure scenario. PFAAs are often highly intercorrelated. It is very diffecult to control for confounding 
by other PFAAs when assessing the effect of single PFAA congeners and interpret the association in light of the high mutual correlation among PFAA congeners. However, given the structural analogy and similar expected modes of action, PFAAs should be regulated as a chemical family rather than isolated compounds [82, 83]. In this regard, the complementation of exposure biomarkers with biomarkers of combined biological activity to PFAS mixtures in epidemiologic studies may be of great help [58]. Berg et al. (2017) [84] did not find an association between summed PFAAs exposure (sum of PFHpS, PFHxS, PFOA, PFOS, PFNA, PFDA and PFUnA) and TH outcome. Whereas, high exposure (4th quartile) to the summed POP (sumPFAAs + sumPCBs [sum of PCB 99,118, 138, 153, 163, 170, 180 and 187] + DDE + $\mathrm{HCB}+$ cis- and trans-nonachlor) was significantly positively associated with maternal TSH [84]. A study found no effect of the summed PFAAs exposure on maternal risk of hypothyroxinaemia (low free $\mathrm{T} 4$ without the compensatory rise in TSH) [85]. Furthermore, one study examined branched isomers of PFOS and found several significant findings, but not with the total PFOS [45].

The selected studies in this review investigated FT4I, FT4, FT3, TT4, TT3, T3RU and TSH in mothers and infants using different methods of chemoluminescent-, radioor enzyme linked immunosorbents assays, with variable sensitivity (e.g. LOD $(\mathrm{TSH})=0.50 \mu \mathrm{U} / \mathrm{mL}$ [38] vs. LOD $(\mathrm{TSH})=0.01 \mu \mathrm{U} / \mathrm{mL}$ [41]). The radio immune sorbent assay seems to be the most reliable method for the quantification of thyroid hormones [86], but all methods showed good sensitivity, specificity, and accuracy. However, the use of immunosassay for hormone determination has been criticized. Animal studies suggested that the effect of PFOS to decrease FT4 level might be due to negative bias in analog techniques resulting from competitive displacement of FT4 and the labeled analog from serum and assay binding proteins in the presence of PFOS [61, 87]. Nevethrthless, such bias was not observed in human study and such different result between human and animal study might be related to the difference of PFAA level, protein binding to T4 (in rats: TTR, in human: TBG) and the interaction of proteins and PFAAs [88]. Based on these reported data, the use of different methods seems not to be of concern in measurements of human thyroid hormones.

Most studies examined $\mathrm{TH}$ at a single time point during pregnancy, impeding the capture of an effect on the thyroidal system during pregnancy since levels vary substantially during different pregnancy stages and stabilises approximately 6 weeks postpartum [34, 89]. However, longitudinal studies measure $\mathrm{TH}$ outcomes multiple times having a greater chance of witnessing an effect on the thyroid system $[42,43,45]$.

TSH was examined in all studies except one [50], and T4 and T3 parameters were less consistently studied.
The free $\mathrm{TH}$ reflects the biologically active hormones, which may give a better estimate of the exposure effect on the thyroid regulatory systems [90]. The FT4I is an estimate of circulating free T4 levels, which accounts for changes in T4 levels due to changes in thyroid binding protein levels and saturation, such as increases in TBG during pregnancy [91], being a better estimate, but only assessed in one single study [39]. Some of the studies only investigated a single $\mathrm{TH}$ parameter $[41,48,50]$, however to understand the effect of PFAA exposure on the $\mathrm{TH}$ equilibrium at least $\mathrm{T} 4$ and $\mathrm{TSH}$ measurements are required.

The extent of adjustment for potential confounders varied largely between studies. General confounders were pre-pregnancy BMI, maternal age, socio-occupational status, parity, alcohol and smoking (Table 2). Eight studies excluded participants with thyroid diseases or related medication $[38,39,41-44,46,48]$ and one study adjusted for these confounders [50]. However, few studies also took into account other thyroid parameters such as iodine status, TPOAb or thyroid binding capacity. Berg et al. demonstrated that several significant effect estimates lost significance after adjusting for thyroid binding capacity in the statistical analysis [43], suggesting an area that should be further studied.

FT4I was suggested as a more reliable FT4 estimate during pregnancy [39]. Weiss et al. found evidence that PFAAs might decrease T4 due to their affinity to thyroid hormone transport proteins using transthyretin (TTR) as carrier protein [55]. However, Preston et al. did not support this theory, after showing lower saturation of plasma binding proteins with higher PFAAs exposure [39]. The incoherent results may be due to the fact that experimental studies use TTR as carrier protein, although the major carrier protein in humans is TBG, which has lower PFAA affinity than TTR [92].

Three studies stratified for TPOAb-status and maternal TSH was associated with several PFAAs in the high TPOAb group [39, 42, 45]. However, the high TPOAb groups differed in sample size (e.g. 14 vs. 98 participants), gestational week (GW) of exposure sampling (median GW 9.6, GW 15-18 and multiple time points), TPO-Ab threshold (Webster et al. and Reardon et al.: > $9 \mathrm{IU} / \mathrm{mL}$ vs. Preston et al.: $>2 \mathrm{U} / \mathrm{mL}$ ) and PFAA concentrations (lower in Reardon et al). Even though results went in different directions, it might indicate a more susceptible group among women with thyroid stress, whom may not be able to compensate for the PFAAs-induced thyroid affections [42]. If these women are highly exposed to PFAAs, which are strongly suspected to affect the thyroid gland, it could increase their risk of immune reactivity and affect the $\mathrm{TH}$ production necessary for a normal foetal development. 


\section{Conclusion}

In overall, most studies supported a positive association of maternal TSH and a possible negative association of maternal T4 and T3 upon PFAAs exposure. Infant $\mathrm{TH}$ might also be affected. The trimester data evaluation showed different direction of associations between PFOS and maternal TSH in different trimester. A positive association between TSH and PFOS was observed in longitudinal studies. The associations of PFAAs and $\mathrm{TH}$ in infant gender differed and were PFAA congeners dependent.

PFAAs exposure has a stronger influence on maternal than infant $\mathrm{TH}$ levels. Scientific evidence seems to favour an increase in maternal TSH and decrease T4 and T3 estimates upon PFAA exposure. The reviewed studies reported some associations between PFAAs and infant TH levels, although these data were less conclusive. The data did not indicate a clear trend in infant gender sensitivity. This review also showed a tendency of trimester dependency of maternal TSH levels, but more studies are needed longitually or during the 1st trimester with follow-up at birth with samples from mother and child (cord blood).

Future studies need a uniform method of examining the associations. We suggest future studies to focus on the 1st trimester with longitially followup in nulliparous women and include a broad range of PFAA congeners.

\section{Supplementary information}

Supplementary information accompanies this paper at https://doi.org/10. 1186/s12940-020-00647-1.

Additional file 1. Association of PFAAs with TSH and TH levels by infant sex.

\begin{abstract}
Abbreviations
BW: Birth weight; BL: Birth length; DDE: Dichlorodiphenyldichloroethylene; EtFOSAA: N-ethyl perfluorooctane sulfonamido acetic acid; 6:2 FTS: 6:2 Fluorotelomer Sulfonate; HC: Head circumference; HCB: Hexachlorbenzen; HPG: Hypothalamic-Pituitary-Gonadal; HPT: Hypothalamic-Pituitary-thyroid; LOD: Limit of detection; LOQ: Limit of quantification; MeFOSAA: 2-(Nmethylperfluorooctanesulfonamido) acetic acid; MESH : Medical Subject Headings; MoA: Mechanism of action; OCPs: Organochlorine pesticides; PCBs: Polychlorinated biphenyls; PFAAs: Perfluorinated-alkyl-acids; PFDA: Perfluorodecanoic acid; PFDoA: Perfluorododecanoic acid; PFHpA: Perfluoroheptanoic acid; PFHpS: Perfluoroheptane sulfonate; PFHXS: Perfluorohexane sulfonate; PFNA: Perfluorononanoic acid; PFOA: Perfluorooctanoate; PFOS: Perfluorooctane sulfonate; PFOSA: Perfluorooctane sulphonamide; PFPeA: Perfluoropentanoic acid; PFUnA: Perfluoroundecanoic acid; PFTrDA: Perfluorotridecanoic acid; PFTeDA: Perfluorotetradecanoic acid; POPs: Persistent organic pollutants; TBG: Thyroxin binding globulin; TH: Thyroid hormones; TSH: Thyroid stimulating hormone; T4: Thyroxine; T3: Triiodothyronine; T3RU: Free triiodothyronine resin uptake; TT4: Total Thyroxine; FT4: Free Thyroxine; FT4I: Free thyroxine index; TPO: Thyroid peroxidase; TPOab: Thyroid peroxidase antibodies; TTR: Transthyretin; UCB: Umbilical cord blood; SGA: Small for gestational age
\end{abstract}

\section{Acknowledgements}

This review is performed as a part of the Human Biomonitoring for Europe (HBM4EU) project, which represents a joint effort of European countries and the European Commission, co-funded by Horizon 2020. The project's main aim is to coordinate and advance human biomonitoring in Europe to provide evidence for policy making. The HBM4EU consortium identified critical questions concerning PFAAs which include the identification of valid effect biomarkers supported on mechanistic pathways.

\section{Authors' contributions}

SB conducted the literature search, constructed tables using data from original manuscripts and was first writing author. MW, ML and EBJ were major contributors of writing the manuscript and proofreading. VM and MF proofread and supported the manuscript. The authors read and approved the final manuscript.

\section{Funding}

This study was supported by the Danish Research Council (30531) and the European Unions' Horizon 2020 research and innovation Programme under grant agreement No 733032 HBM4EU (www.HBM4EU.eu).

\section{Availability of data and materials}

Not applicable. No datasets were generated or analyzed during the current study. All original manuscripts are available online at https://www.ncbi.nlm. nih.gov/pubmed/

Ethics approval and consent to participate

Not applicable.

\section{Consent for publication}

Not applicable.

\section{Competing interests}

The authors declare that they have no competing interests.

\section{Author details}

${ }^{1}$ Centre for Arctic Health and Molecular Epidemiology, Department of Public Health, Aarhus University, Aarhus, Denmark. ${ }^{2}$ School of Medicine, Center of Biomedical Research, University of Granada, Granada, Spain. ${ }^{3}$ Consortium for Biomedical Research in Epidemiology \& Public Health (CIBERESP), Madrid, Spain. ${ }^{4}$ Greenland Centre for Health Research, University of Greenland, Nuuk, Greenland.

Received: 29 November 2019 Accepted: 19 August 2020

Published online: 13 October 2020

\section{References}

1. Rappazzo KM, Coffman E, Hines EP. Exposure to Perfluorinated Alkyl Substances and Health Outcomes in Children: A Systematic Review of the Epidemiologic Literature. Int J Environ Res Public Health. 2017;14(7):691. https://doi.org/10.3390/ijerph14070691.

2. Weihe P, Debes F, Halling J, Petersen MS, Muckle G, Odland JO, et al. Health effects associated with measured levels of contaminants in the Arctic. Int $J$ Circumpolar Health. 2016;75:33805.

3. Gilbert ME, Rovet J, Chen Z, Koibuchi N. Developmental thyroid hormone disruption: prevalence, environmental contaminants and neurodevelopmental consequences. Neurotoxicology. 2012:33(4):842-52.

4. Pessah IN, Lein PJ, Seegal RF, Sagiv SK. Neurotoxicity of polychlorinated biphenyls and related organohalogens. Acta Neuropathol. 2019;138(3):363-87.

5. Heilmann C, Budtz-Jørgensen E, Nielsen F, Heinzow B, Weihe P, Grandjean $P$. Serum concentrations of antibodies against vaccine toxoids in children exposed perinatally to Immunotoxicants. Environ Health Perspect. 2010; 118(10):1434-8.

6. Grandjean P, Andersen EW, Budtz-Jørgensen E, Nielsen F, Mølbak K, Weihe $P$, et al. Serum vaccine antibody concentrations in children exposed to Perfluorinated compounds. JAMA. 2012;307(4):391-7.

7. Wielsoe M, Kern P, Bonefeld-Jorgensen EC. Serum levels of environmental pollutants is a risk factor for breast cancer in Inuit: a case control study. Environ Health. 2017;16(1):56

8. Sun Q, Zong G, Valvi D, Nielsen F, Coull B, Grandjean P. Plasma concentrations of Perfluoroalkyl substances and risk of type 2 diabetes: a prospective investigation among U.S. women. Environ Health Perspect. 2018;126(3):037001. 
9. Bjerregaard P. Cardiovascular disease and environmental pollutants: the Arctic aspect. Arctic Med Res. 1996;55(Suppl 1):25-31.

10. Sergeev AV, Carpenter DO. Hospitalization rates for coronary heart disease in relation to residence near areas contaminated with persistent organic pollutants and other pollutants. Environ Health Perspect. 2005;113(6):756-61.

11. Kannan K, Koistinen J, Beckmen K, Evans T, Gorzelany JF, Hansen KJ, et al. Accumulation of perfluorooctane sulfonate in marine mammals. Environ Sci Technol. 2001;35(8):1593-8.

12. Giesy JP, Kannan K. Global distribution of perfluorooctane sulfonate in wildlife. Environ Sci Technol. 2001;35(7):1339-42.

13. Bjerregaard-Olesen C, Bossi R, Liew Z, Long M, Bech BH, Olsen J, et al. Maternal serum concentrations of perfluoroalkyl acids in five international birth cohorts. Int J Hyg Environ Health. 2017;220(2 Pt A):86-93.

14. Shoeib M, Harner T. G MW, Lee SC: indoor sources of poly- and perfluorinated compounds (PFCS) in Vancouver, Canada: implications for human exposure. Environ Sci Technol. 2011;45(19):7999-8005.

15. Olsen GW, Burris JM, Ehresman DJ, Froehlich JW, Seacat AM, Butenhoff JL, et al. Half-life of serum elimination of

perfluorooctanesulfonate,perfluorohexanesulfonate, and perfluorooctanoate in retired fluorochemical production workers. Environ Health Perspect. 2007; 115(9):1298-305.

16. REACH. Regulation (EC) no 1907/2006 of the European Parliament and of the council of 18 December 2006 concerning the registration, evaluation, authorisation and restriction of chemicals (REACH). Off J EU. 2006; L396(2006):1-849.

17. Stockholm convention listing of POPs in the Stockholm Convention [http:// chm.pops.int/Convention/ThePOPs/ListingofPOPs/tabid/2509/Default.aspx]. Accessed 10 June 2020.

18. Bjerregaard-Olesen C, Bach CC, Long M, Ghisari M, Bossi R, Bech BH, et al. Time trends of perfluorinated alkyl acids in serum from Danish pregnant women 2008-2013. Environ Int. 2016;91:14-21.

19. Glynn A, Berger U, Bignert A, Ullah S, Aune M, Lignell S, et al. Perfluorinated alkyl acids in blood serum from primiparous women in Sweden: serial sampling during pregnancy and nursing, and temporal trends 1996-2010. Environ Sci Technol. 2012;46(16):9071-9.

20. Fromme H, Mosch C, Morovitz M, Alba-Alejandre I, Boehmer S, Kiranoglu M, et al. Pre- and postnatal exposure to perfluorinated compounds (PFCs). Environ Sci Technol. 2010;44(18):7123-9.

21. Yang L, Wang Z, Shi Y, Li J, Wang Y, Zhao Y, et al. Human placental transfer of perfluoroalkyl acid precursors: levels and profiles in paired maternal and cord serum. Chemosphere. 2016;144:1631-8.

22. Boas M, Feldt-Rasmussen U, Main KM. Thyroid effects of endocrine disrupting chemicals. Mol Cell Endocrinol. 2012;355(2):240-8.

23. de Escobar GM, Obregon MJ, del Rey FE. Maternal thyroid hormones early in pregnancy and fetal brain development. Best Pract Res Clin Endocrinol Metab. 2004;18(2):225-48.

24. Morreale de Escobar G, Obregon MJ, Escobar del Rey F. Role of thyroid hormone during early brain development. Eur J Endocrinol. 2004;151(Suppl 3):U25-37. https://doi.org/10.1530/eje.0.151u025.

25. Andersen SL, Olsen J, Wu CS, Laurberg P. Low birth weight in children born to mothers with hyperthyroidism and high birth weight in hypothyroidism, whereas preterm birth is common in both conditions: a Danish National Hospital Register Study. Eur Thyroid J. 2013;2(2):135-44.

26. Krassas GE, Poppe K, Glinoer D. Thyroid function and human reproductive health. Endocr Rev. 2010;31(5):702-55.

27. Thompson W, Russell G, Baragwanath G, Matthews J, Vaidya B, ThompsonCoon J. Maternal thyroid hormone insufficiency during pregnancy and risk of neurodevelopmental disorders in offspring: a systematic review and meta-analysis. Clin Endocrinol (Oxf). 2018;88(4):575-84.

28. Maraka S, Ospina NM, O'Keeffe DT, Espinosa De Ycaza AE, Gionfriddo MR, Erwin PJ, et al. Subclinical hypothyroidism in pregnancy: a systematic review and meta-analysis. Thyroid. 2016;26(4):580-90.

29. Leung AM. Thyroid function in pregnancy. J Trace Elem Med Biol. 2012; 26(2-3):137-40.

30. Roti E, Gnudi A, Braverman LE. The placental transport, synthesis and metabolism of hormones and drugs which affect thyroid function*. Endocr Rev. 1983;4(2):131-49.

31. Laurberg P, Andersen SL, Hindersson P, Nohr EA, Olsen J. Dynamics and predictors of serum TSH and fT4 reference limits in early pregnancy: a study within the Danish National Birth Cohort. J Clin Endocrinol Metabol. 2016; 101(6):2484-92.
32. Lazarus J, Brown RS, Daumerie C, Hubalewska-Dydejczyk A, Negro R, Vaidya B. 2014 European thyroid association guidelines for the Management of Subclinical Hypothyroidism in pregnancy and in children. European Thyroid J. 2014;3(2):76-94.

33. Alexander EK, Pearce EN, Brent GA, Brown RS, Chen H, Dosiou C, et al. 2017 guidelines of the American Thyroid Association for the diagnosis and Management of Thyroid Disease during pregnancy and the postpartum. Thyroid. 2017;27(3):315-89.

34. Glinoer D, de Nayer $P$, Bourdoux $P$, Lemone $M$, Robyn $C$, van Steirteghem A, et al. Regulation of maternal thyroid during pregnancy. J Clin Endocrinol Metab. 1990;71(2):276-87.

35. NBV: Thyroideasygdom ved graviditet og infertiliet. http://www. endocrinology.dk/index.php/nbvhovedmenu/2-thyroidea-sygdomme/5thyroideasygdom-ved-graviditet-og-infertilitet\#anchor1. Accessed 27 Aug 2020.

36. Feldt-Rasmussen U, Petersen PH, Blaabjerg O, Hørder M. Long-term variability in serum thyroglobulin and thyroid related hormones in healthy subjects. Acta Endocrinol. 1980;95(3):328-34.

37. Song M, Kim YJ, Park YK, Ryu JC. Changes in thyroid peroxidase activity in response to various chemicals. J Environ Monit. 2012;14(8):2121-6.

38. Kato S, Itoh S, Yuasa M, Baba T, Miyashita C, Sasaki S, et al. Association of perfluorinated chemical exposure in utero with maternal and infant thyroid hormone levels in the Sapporo cohort of Hokkaido study on the environment and Children's health. Environ Health Prev Med. 2016;21(5): 334-44.

39. Preston EV, Webster TF, Oken E, Claus Henn B, McClean MD, Rifas-Shiman SL, et al. Maternal plasma per- and Polyfluoroalkyl substance concentrations in early pregnancy and maternal and neonatal thyroid function in a prospective birth cohort: project viva (USA). Environ Health Perspect. 2018; 126(2):027013.

40. Inoue K, Ritz B, Andersen SL, Ramlau-Hansen CH, Hoyer BB, Bech BH, et al. Perfluoroalkyl substances and maternal thyroid hormones in early pregnancy; findings in the Danish national birth cohort. Environ Health Perspect. 2019;127(11):117002.

41. Wang Y, Starling AP, Haug LS, Eggesbo M, Becher G, Thomsen C, et al. Association between perfluoroalkyl substances and thyroid stimulating hormone among pregnant women: a cross-sectional study. Environ Health. 2013;12(1):76.

42. Webster GM, Venners SA, Mattman A, Martin JW. Associations between perfluoroalkyl acids (PFASs) and maternal thyroid hormones in early pregnancy: a population-based cohort study. Environ Res. 2014;133:338-47.

43. Berg V, Nost TH, Hansen S, Elverland A, Veyhe AS, Jorde R, et al. Assessing the relationship between perfluoroalkyl substances, thyroid hormones and binding proteins in pregnant women; a longitudinal mixed effects approach. Environ Int. 2015;77:63-9.

44. Wang Y, Rogan WJ, Chen PC, Lien GW, Chen HY, Tseng YC, et al. Association between maternal serum perfluoroalkyl substances during pregnancy and maternal and cord thyroid hormones: Taiwan maternal and infant cohort study. Environ Health Perspect. 2014;122(5):529-34.

45. Reardon AJF, Khodayari Moez E, Dinu I, Goruk S, Field CJ, Kinniburgh DW, et al. Longitudinal analysis reveals early-pregnancy associations between perfluoroalkyl sulfonates and thyroid hormone status in a Canadian prospective birth cohort. Environ Int. 2019;129:389-99.

46. Yang L, Li J, Lai J, Luan H, Cai Z, Wang Y, et al. Placental transfer of Perfluoroalkyl substances and associations with thyroid hormones: Beijing prenatal exposure study. Sci Rep. 2016;6:21699.

47. Xiao C, Grandjean P, Valvi D, Nielsen F, Jensen TK, Weihe P, et al. Associations of exposure to perfluoroalkyl substances with thyroid hormone concentrations and birth size. J Clin Endocrinol Metab. 2020;105(3):dgz147. https://doi.org/10.1210/clinem/dgz147.

48. Dufour P, Pirard C, Seghaye MC, Charlier C. Association between organohalogenated pollutants in cord blood and thyroid function in newborns and mothers from Belgian population. Environ Pollut. 2018;238: 389-96.

49. Shah-Kulkarni S, Kim BM, Hong YC, Kim HS, Kwon EJ, Park H, et al. Prenatal exposure to perfluorinated compounds affects thyroid hormone levels in newborn girls. Environ Int. 2016;94:607-13.

50. de Cock M, de Boer MR, Lamoree M, Legler J, van de Bor M. Prenatal exposure to endocrine disrupting chemicals in relation to thyroid hormone levels in infants - a Dutch prospective cohort study. Environ Health. 2014;13:106. 
51. Tsai MS, Lin CC, Chen MH, Hsieh WS, Chen PC. Perfluoroalkyl substances and thyroid hormones in cord blood. Environ Pollut. 2017;222:543-8.

52. Aimuzi R, Luo K, Chen Q, Wang H, Feng L, Ouyang F, et al. Perfluoroalkyl and polyfluoroalkyl substances and fetal thyroid hormone levels in umbilical cord blood among newborns by prelabor caesarean delivery. Environ Int. 2019;130:104929.

53. Ballesteros V, Costa O, Iniguez C, Fletcher T, Ballester F, Lopez-Espinosa MJ. Exposure to perfluoroalkyl substances and thyroid function in pregnant women and children: a systematic review of epidemiologic studies. Environ Int. 2017:99:15-28.

54. Kabir ER, Rahman MS, Rahman I. A review on endocrine disruptors and their possible impacts on human health. Environ Toxicol Pharmacol. 2015;40(1):241-58.

55. Weiss JM, Andersson PL, Lamoree MH, Leonards PEG, van Leeuwen SPJ, Hamers T. Competitive binding of poly- and Perfluorinated compounds to the thyroid hormone transport protein transthyretin. Toxicol Sci. 2009;109(2):206-16.

56. Yu W-G, Liu W, Jin Y-H. Effects of perfluorooctane sulfonate on rat thyroid hormone biosynthesis and metabolism. Environ Toxicol Chem. 2009;28(5):990-6.

57. Long M, Ghisari M, Bonefeld-Jorgensen EC. Effects of perfluoroalkyl acids on the function of the thyroid hormone and the aryl hydrocarbon receptor. Environ Sci Pollut Res Int. 2013;20(11):8045-56.

58. Bjerregaard-Olesen C, Bach CC, Long M, Wielsoe M, Bech BH, Henriksen TB, et al. Associations of fetal growth outcomes with measures of the combined Xenoestrogenic activity of maternal serum Perfluorinated alkyl acids in Danish pregnant women. Environ Health Perspect. 2019;127(1):17006.

59. Lau C, Anitole K, Hodes C, Lai D, Pfahles-Hutchens A, Seed J. Perfluoroalkyl acids: a review of monitoring and toxicological findings. Toxicol Sci. 2007;99(2):366-94.

60. Thibodeaux JR, Hanson RG, Rogers JM, Grey BE, Barbee BD, Richards JH, et al. Exposure to perfluorooctane sulfonate during pregnancy in rat and mouse. I: maternal and prenatal evaluations. Toxicol Sci. 2003;74(2):369-81.

61. Luebker DJ, York RG, Hansen KJ, Moore JA, Butenhoff JL. Neonatal mortality from in utero exposure to perfluorooctanesulfonate (PFOS) in SpragueDawley rats: dose-response, and biochemical and pharamacokinetic parameters. Toxicology. 2005;215(1-2):149-69.

62. Koustas E, Lam J, Sutton P, Johnson PI, Atchley DS, Sen S, et al. The navigation guide - evidence-based medicine meets environmental health: systematic review of nonhuman evidence for PFOA effects on fetal growth. Environ Health Perspect. 2014;122(10):1015-27.

63. Chang SC, Ehresman DJ, Bjork JA, Wallace KB, Parker GA, Stump DG, et al. Gestational and lactational exposure to potassium perfluorooctanesulfonate (K+PFOS) in rats: toxicokinetics, thyroid hormone status, and related gene expression. Reprod Toxicol. 2009;27(3-4):387-99.

64. Lau C, Thibodeaux JR, Hanson RG, Rogers JM, Grey BE, Stanton ME, et al. Exposure to perfluorooctane sulfonate during pregnancy in rat and mouse. II: postnatal evaluation. Toxicol Sci. 2003;74(2):382-92.

65. Seacat AM, Thomford PJ, Hansen KJ, Olsen GW, Case MT, Butenhoff JL. Subchronic toxicity studies on perfluorooctanesulfonate potassium salt in cynomolgus monkeys. Toxicol Sci. 2002;68(1):249-64.

66. Wei Y, Dai J, Liu M, Wang J, Xu M, Zha J, et al. Estrogen-like properties of perfluorooctanoic acid as revealed by expressing hepatic estrogenresponsive genes in rare minnows (Gobiocypris rarus). Environ Toxicol Chem. 2007;26(11):2440-7.

67. Olsen GW, Butenhoff JL, Zobel LR. Perfluoroalkyl chemicals and human fetal development: an epidemiologic review with clinical and toxicological perspectives. Reprod Toxicol. 2009;27(3-4):212-30.

68. Ghisari M, Bonefeld-Jorgensen EC. Impact of environmental chemicals on the thyroid hormone function in pituitary rat GH3 cells. Mol Cell Endocrinol. 2005;244(1-2):31-41

69. Bonefeld-Jorgensen EC, Ghisari M, Wielsoe M, Bjerregaard-Olesen C, Kjeldsen LS, Long M. Biomonitoring and hormone-disrupting effect biomarkers of persistent organic pollutants in vitro and ex vivo. Basic Clin Pharmaco Toxicol. 2014;115(1):118-28.

70. Kjeldsen LS, Bonefeld-Jorgensen EC. Perfluorinated compounds affect the function of sex hormone receptors. Environ Sci Pollut Res Int. 2013;20(11):8031-44.

71. Kraugerud M, Zimmer KE, Ropstad E, Verhaegen S. Perfluorinated compounds differentially affect steroidogenesis and viability in the human adrenocortical carcinoma (H295R) in vitro cell assay. Toxicol Lett. 2011;205(1):62-8.

72. Bjerregaard-Olesen C, Bossi R, Bech BH, Bonefeld-Jorgensen EC. Extraction of perfluorinated alkyl acids from human serum for determination of the combined xenoestrogenic transactivity: a method development. Chemosphere. 2015;129:232-8.
73. Bjerregaard-Olesen C, Ghisari M, Bonefeld-Jorgensen EC. Activation of the estrogen receptor by human serum extracts containing mixtures of perfluorinated alkyl acids from pregnant women. Environ Res. 2016;151:71-9.

74. Aylward LL, Hays SM, Kirman CR, Marchitti SA, Kenneke JF, English C, et al. Relationships of chemical concentrations in maternal and cord blood: a review of available data. J Toxicol Environ Health B Crit Rev. 2014;17(3):175-203.

75. Monroy R, Morrison K, Teo K, Atkinson S, Kubwabo C, Stewart B, et al. Serum levels of perfluoroalkyl compounds in human maternal and umbilical cord blood samples. Environ Res. 2008;108(1):56-62.

76. Fei C, McLaughlin JK, Tarone RE, Olsen J. Perfluorinated chemicals and fetal growth: a study within the Danish national birth cohort. Environ Health Perspect. 2007;115(11):1677-82.

77. Sagiv SK, Rifas-Shiman SL, Fleisch AF, Webster TF, Calafat AM, Ye X, et al. Early-pregnancy plasma concentrations of Perfluoroalkyl substances and birth outcomes in project viva: confounded by pregnancy hemodynamics? Am J Epidemiol. 2018;187(4):793-802.

78. Harada K, Inoue K, Morikawa A, Yoshinaga T, Saito N, Koizumi A. Renal clearance of perfluorooctane sulfonate and perfluorooctanoate in humans and their species-specific excretion. Environ Res. 2005;99(2):253-61.

79. Gutzkow KB, Haug LS, Thomsen C, Sabaredzovic A, Becher G, Brunborg G. Placental transfer of perfluorinated compounds is selective--a Norwegian mother and child sub-cohort study. Int J Hyg Environ Health. 2012;215(2):216-9.

80. Haug LS, Huber S, Becher G, Thomsen C. Characterisation of human exposure pathways to perfluorinated compounds--comparing exposure estimates with biomarkers of exposure. Environ Int. 2011;37(4):687-93.

81. Fisher M, Arbuckle TE, Liang CL, LeBlanc A, Gaudreau E, Foster WG, et al. Concentrations of persistent organic pollutants in maternal and cord blood from the maternal-infant research on environmental chemicals (MIREC) cohort study. Environ Health. 2016;15(1):59.

82. Examining the Federal response to the risks associated with per- and polyfluoroalkyl sub-stances (PFAS). Senate Committe on Environment and Public Works: National Institute of Environmen-tal Health Sciences [https:// www.epw.senate.gov/public/_cache/files/2/2/22ca7c4b-b1dc-4a12-9264-7a4 f16608933/BF2D70A4FB747A3F61E584CC30D58D0A.birnbaum-testimony03.28.2019.pdf].

83. Birnbaum LS, Grandjean P. Alternatives to PFASs: perspectives on the science. Environ Health Perspect. 2015;123(5):A104-5.

84. Berg V, Nost TH, Pettersen RD, Hansen S, Veyhe AS, Jorde R, et al. Persistent organic pollutants and the association with maternal and infant thyroid homeostasis: a multipollutant assessment. Environ Health Perspect. 2017; 125(1):127-33.

85. Chan E, Burstyn I, Cherry N, Bamforth F, Martin JW. Perfluorinated acids and hypothyroxinemia in pregnant women. Environ Res. 2011;111(4):559-64.

86. Shamsian AA, Ghazvini K, Sokhtanloo M, Moghaddam MS, Vakili R. Which quantitative method in determination of the thyroid hormone levels is more consistent with the clinical symptoms of the thyroid disorders? Comp Clin Pathol. 2016;25(1):101-6.

87. Chang SC, Thibodeaux JR, Eastvold ML, Ehresman DJ, Bjork JA, Froehlich JW, et al. Negative bias from analog methods used in the analysis of free thyroxine in rat serum containing perfluorooctanesulfonate (PFOS). Toxicology. 2007;234(1-2):21-33.

88. Lopez-Espinosa MJ, Fitz-Simon N, Bloom MS, Calafat AM, Fletcher T. Comparison between free serum thyroxine levels, measured by analog and dialysis methods, in the presence of perfluorooctane sulfonate and perfluorooctanoate. Reprod Toxicol. 2012;33(4):552-5.

89. Hoover C. Maternal, fetal, \& neonatal physiology: a clinical perspective2nd edition By Susan Tucker Blackburn. St. Louis, MO: Saunders, 2003. 781 pages. $\$ 99.00$, hardcover. J Midwifery Womens Health. 2004;49(2):164-5.

90. Fitzpatrick DL, Russell MA. Diagnosis and management of thyroid disease in pregnancy. Obstet Gynecol Clin North Am. 2010;37(2):173-93.

91. Lee RH, Spencer CA, Mestman JH, Miller EA, Petrovic I, Braverman LE, et al. Free T4 immunoassays are flawed during pregnancy. Am J Obstet Gynecol. 2009;200(3):260.e261-6.

92. Ren X-M, Qin W-P, Cao L-Y, Zhang J, Yang Y, Wan B, et al. Binding interactions of perfluoroalkyl substances with thyroid hormone transport proteins and potential toxicological implications. Toxicology. 2016;366-367:32-42.

\section{Publisher's Note}

Springer Nature remains neutral with regard to jurisdictional claims in published maps and institutional affiliations. 